\title{
Optical trapping and manipulation of plasmonic nanoparticles: fundamentals, applications, and perspectives
}

\author{
Alexander S. Urban, $\uparrow$ Sol Carretero-Palacios, Andrey A. Lutich, Theobald Lohmüller, \\ Jochen Feldmann and Frank Jäckelț*
}

Received 13th December 2013

Accepted 6th March 2014

DOI: $10.1039 / \mathrm{c} 3 \mathrm{nr} 06617 \mathrm{~g}$

www.rsc.org/nanoscale

\begin{abstract}
This feature article discusses the optical trapping and manipulation of plasmonic nanoparticles, an area of current interest with potential applications in nanofabrication, sensing, analytics, biology and medicine. We give an overview over the basic theoretical concepts relating to optical forces, plasmon resonances and plasmonic heating. We discuss fundamental studies of plasmonic particles in optical traps and the temperature profiles around them. We place a particular emphasis on our own work employing optically trapped plasmonic nanoparticles towards nanofabrication, manipulation of biomimetic objects and sensing.
\end{abstract}

\section{Introduction}

The ability of light to exert mechanical forces on objects that it interacts with was first hypothesised by Kepler in 1619 to explain why comets' tails always point away from the sun. ${ }^{1}$ This was likely motivated by earlier descriptions of Halley's comet by Apian in $1532 .{ }^{2}$ The theoretical underpinning of these forces, i.e. the radiation pressure, was provided by Maxwell's theory of electromagnetism in the 19th century. ${ }^{3}$ Experimental verification of the radiation pressure was provided by Lebedew as well as Fox and Hull around the following turn of the century, by measuring the movement of torsion pendulums under illumination. ${ }^{4,5}$ In a particle picture, the radiation pressure can be understood as a consequence of the conservation of momentum during absorption and scattering of photons. In addition to this scattering force, electromagnetic fields induce a polarisation in dielectric materials which results in a gradient force pointing towards high intensity field regions. It was this gradient force of a single tightly focused laser beam that allowed Ashkin $e t$ al. in 1986 to trap individual nano- and microsized dielectric particles in three dimensions. ${ }^{6}$ This technique has since become known as optical tweezers and has found wide-spread applications in biology, physics, chemistry and materials science since it allows applying well-defined forces and torques and manipulating nano- and microscale objects non-invasively. ${ }^{7-9}$

Photonics and Optoelectronics Group, Department of Physics and Center for NanoScience (CeNS), Ludwig-Maximilians-Universität München, Amalienstr. 54, 80799 Munich, Germany. E-mail: fjaeckel@liv.ac.uk

$\dagger$ Present address: Laboratory for Nanophotonics, Rice University, Department of Electrical and Computer Engineering, 6100 Main Street, Houston, TX 77005, USA. \$ Present address: Department of Physics and Stephenson Institute for Renewable Energy, University of Liverpool, Chadwick Building, Peach Street, L69 7ZF, Liverpool, UK.
Optical tweezers for dielectric particles such as latex or silica spheres are dominated by the gradient force. Metallic nanoparticles on the other hand strongly absorb and scatter light close to their localised surface plasmon resonance (LSPR), which increases the relative contribution of the scattering force and makes stable trapping more difficult. However, Svoboda and Block showed in 1994 that metallic nanoparticles can be stably trapped with sufficiently off-resonant optical tweezers (i.e. a focused laser with a wavelength not too close to the LSPR of the trapped particle)..$^{10}$ This has opened the door to applying. the extraordinary properties of plasmonic particles in free solution and in absence of a substrate. ${ }^{11,12}$ These properties include electrical field enhancement, enhancement of fluorescence and Raman scattering as well as local heating. In addition, the subtle balance between gradient and scattering force, which can be achieved near the LSPR, allows for precise manipulation of trapped particles. This combination of precise spatial control and plasmonic properties renders optical trapping and manipulation of plasmonic particles important for a wide range of research fields, both fundamental and applied. All-optical nanofabrication techniques based on plasmonic particle trapping and manipulation, for instance, may find applications for flexible design and fabrication of plasmonic sensors for detection of pollutants, analytes or biological markers in environmental, chemical or medical applications. ${ }^{13}$ They also may allow fabrication of nanoplasmonic structures for future optical and optoelectronic circuitry, and structuring of polymers for nanofluidics. ${ }^{14,15}$ In combination with biological objects, novel approaches to drug delivery and cellular manipulation may be enabled. ${ }^{16}$ In addition, fundamental studies of, e.g. plasmonic heating are beneficial for cancer treatment applications. ${ }^{17}$ Many of the above applications are in their early or proof-of-principle stages; a fact that provides ample room for future research. 
This feature article discusses recent developments in the optical trapping and manipulation of plasmonic particles. While we highlight important contributions from other groups we will focus on the discussion of our own work. The remainder of this article is organized as follows: after this short general introduction we will review the basic properties of optical forces and plasmonic nanoparticles that are the foundation of the subsequently discussed studies. This is followed by a discussion of experimental studies into the fundamental aspects of plasmonic particles in optical traps. Before an outlook is given, we discuss more applied experiments with optically trapped plasmonic nanoparticles that aim at nanofabrication, manipulation of bio-mimetic structures, and sensing.

\section{Basics and modelling}

In this section we summarise the theoretical concepts related to optical trapping of plasmonic nanoparticles, such as polarisability, scattering and absorption cross sections, optical forces, and local heating, providing the main equations to calculate these quantities and the range of their validity. References for full derivations will be provided where appropriate.

\section{Plasmon resonances}

The optical properties of noble metals can be described by a complex dielectric function $\varepsilon_{\mathrm{m}}(\omega)$ that depends on the frequency of the incident light $(\omega)$. There are two major contributions to $\varepsilon_{\mathrm{m}}(\omega):{ }^{18}$ (i) the quasi-free movement of the conduction band electrons within the bulk material induced by the incident radiation, and (ii) the electronic interband transitions that may take place if the energy of the incoming photons exceeds the energy between different bands. The dielectric function containing both contributions can be described by the sum of Drude and Lorentzian terms ( $j$ ) in the Drude-Lorentz model:

$$
\varepsilon_{\mathrm{m}}(\omega)=\varepsilon_{\mathrm{r}}-\sum_{j} \frac{\omega_{\mathrm{P}_{j}}{ }^{2}}{\omega\left(\omega+i \gamma_{j}\right)}+\sum_{j} \frac{\Delta \varepsilon_{j} \Omega_{\mathrm{P}_{j}}{ }^{2}}{\Omega_{\mathrm{P}_{j}}{ }^{2}-\omega^{2}-i \Gamma_{j} \omega} .
$$

In the Drude term, $\varepsilon_{\mathrm{r}}$ describes the optical response at high frequencies, which includes the background dielectric function of the ionic cores, $\omega_{\mathrm{P}}$ is the volume plasma frequency, and $\gamma$ is the damping time related to energy losses by heating. In the Lorentzian term, $\Delta \varepsilon$ weights the contribution of the given interband transition to the dielectric function and $\Omega_{\mathrm{p}}$ and $\Gamma$ are the plasma and damping frequencies for the bound electrons, respectively. The inclusion of more terms in the sum provides a better fitting at short wavelengths $(\sim 400 \mathrm{~nm})$ to the experimental data. ${ }^{19-23}$ The values of fitting parameters for different metals $(\mathrm{Ag}, \mathrm{Au}, \mathrm{Cu}$, $\mathrm{Al}$, Ni and others) can be found in literature. ${ }^{21,22,24,25}$

Upon illumination, a nanoparticle both absorbs and scatters light, which leads to attenuation (i.e. extinction) of the incident electromagnetic (EM) wave. These processes are described by scattering, absorption, and extinction cross sections. The calculation of the EM fields at all points in space in the general case when light interacts with a particle of arbitrary shape and dielectric properties is a complex problem that must be solved numerically. ${ }^{\text {26-37 }}$ However, simple cases can be treated analytically and provide important insights. The quasi-static approximation, for instance, neglects retardation effects, i.e., all points of an object respond simultaneously to an incoming (excitation) field. ${ }^{38}$ This approximation is valid only if the size of the object is much smaller than the wavelength of light (Rayleigh particle). ${ }^{39,40}$

We first consider a metallic sphere of radius $a$ much smaller than the incident wavelength in vacuum $(a \ll \lambda)$ embedded in a medium with dielectric constant $\varepsilon$ in a uniform, static electric field. This system is well described by a dipole with dipole moment $\vec{p}=\varepsilon_{0} \varepsilon \alpha(\omega) \vec{E}_{0}$ induced by the external field $\left(\vec{E}_{0}\right) . \varepsilon_{0}$ is the permittivity of free space, and $\alpha(\omega)$ denotes the complex polarisability of a Rayleigh sphere in the electrostatic approximation: ${ }^{18,39-42}$

$$
\alpha(\omega)=3 V \frac{\varepsilon_{\mathrm{m}}(\omega)-\varepsilon}{\varepsilon_{\mathrm{m}}(\omega)+2 \varepsilon},
$$

with $V=\frac{4 \pi a^{3}}{3}$ the volume of the sphere. $\alpha(\omega)$ already describes the dipolar surface plasmon, i.e. a resonance for minimal values of $\left|\varepsilon_{\mathrm{m}}(\omega)+2 \varepsilon\right|$, when $\operatorname{Re}\left[\varepsilon_{\mathrm{m}}(\omega)\right]=-2 \varepsilon^{\mathbf{1 8 , 4 2}}$ This description predicts that the nanoparticle acts as an electric dipole, resonantly absorbing and scattering EM fields at its resonance. This is the simplest way to determine the resonance wavelength of a spherical metal nanoparticle with dimensions below some tens of nanometres $(a \ll \lambda)$, embedded in media with low dielectric constant, in the visible and near-infrared regimes. However, within this approximation the resonance wavelength of a particle within a given medium depends only on its material, and the experimentally observed red-shift and broadening of the resonance with increasing particle size is not captured by the model.

For larger particles with $a \approx \lambda$, a rigorous electrodynamics approach is needed. Mie theory is the most popular model to describe the scattering process of a small particle, which incorporates the contribution of higher multipolar orders. ${ }^{43} \mathrm{~A}$ full description of Mie theory can be found elsewhere. ${ }^{38,44}$ Here, we will limit ourselves to extending the quasi-static approximation to particles with dimensions $a \approx \lambda$. Within this approach, the retardation effects are considered by an expansion of the first TM mode of Mie's formulation, providing an analytical formula that allows one to gain useful physical insight: ${ }^{\mathbf{4 5 , 4 6}}$

$$
\alpha(\omega)=V \frac{1-(1 / 10)\left(\varepsilon_{\mathrm{m}}(\omega)+\varepsilon\right) x^{2}+O\left(x^{4}\right)}{\left(\frac{1}{3}+\frac{\varepsilon}{\varepsilon_{\mathrm{m}}(\omega)-\varepsilon}\right)-\frac{1}{30}\left(\varepsilon_{\mathrm{m}}(\omega)+10 \varepsilon\right) x^{2}-i \frac{4 \pi^{2} \varepsilon^{3 / 2}}{3} \frac{V}{\lambda^{3}}+O\left(x^{4}\right)} .
$$

In the above equation, $x=\frac{2 \pi a}{\lambda}$ is the size parameter and $O\left(x^{4}\right)$ contains higher orders of the size parameter. Eqn (3) provides the complex polarisability of metal nanoparticles with dimensions $a \approx \lambda$, describing the red-shift of the plasmon resonance with increasing particle size. Generalizations of eqn (3) for non-spherical objects, ellipsoids, and spheroids have been proposed. ${ }^{\mathbf{4 4 , 4 5}}$ Although it is easy to calculate $\alpha(\omega)$ as shown in eqn (3), there is no easy way to measure it experimentally 
since one must determine the charge distribution of the entire metal sphere upon light excitation.

Quantities that are experimentally accessible are the scattering and extinction cross sections of plasmonic particles, which describe the ratio between the scattered (or extinct) power and the incident light intensity: ${ }^{38,42}$

$$
\begin{aligned}
& \sigma_{\text {scatt }}=\frac{k^{4}}{6 \pi}|\alpha(\omega)|^{2}, \\
& \sigma_{\text {ext }}=k \alpha^{\prime}(\omega),
\end{aligned}
$$

with $k=2 \pi \sqrt{\varepsilon} / \lambda$, and $\alpha^{\prime}(\omega)$ the real part of the complex polarisability $\alpha(\omega)=\alpha^{\prime}(\omega)+i \alpha^{\prime \prime}(\omega)$. Since extinction is the combination of scattering and absorption, the absorption cross section can be obtained from $\sigma_{\text {ext }}=\sigma_{\text {scatt }}+\sigma_{\text {abs }}$. Fig. 1a displays the exact Finite-Difference Time-Domain (FDTD) calculations of $\sigma_{\text {ext }}, \sigma_{\text {scatt }}$ and $\sigma_{\text {abs }}$ of a gold nanoparticle of $a=40 \mathrm{~nm}$ in water $(\varepsilon=1.8)$, and a comparison with the analytical description after combining eqn (1) and (3) into (4). The agreement of both the resonance wavelength and magnitudes between the two methods is excellent. Fig. 1b displays the real $\left(\alpha^{\prime}\right)$ and imaginary $\left(\alpha^{\prime \prime}\right)$ parts of the polarisability calculated for the same system using eqn (3). It can be seen that the plasmon resonance at $\lambda=570 \mathrm{~nm}$ (Fig. 1a) corresponds to resonances in $\alpha(\omega)$.

\section{Optical forces}

The force acting on a nanoparticle when illuminated by a laser beam will depend on the characteristics of both the laser and the nanoparticle. Of particular importance is whether or not the laser is resonant with the LSPR of the particle. The time averaged force $\langle\vec{F}\rangle$ on a particle due to harmonic fields may be calculated from the Maxwell's stress tensor, ${ }^{18} \stackrel{\leftrightarrow}{T}$. The net force can be found by integrating Maxwell's stress tensor over a closed surface surrounding the particle $(\partial V)$ :

$$
\langle\vec{F}\rangle=\int_{\partial V}\langle\stackrel{\leftrightarrow}{T}(\vec{r}, t)\rangle \vec{n}(\vec{r}) \mathrm{d} a
$$

where $\langle\cdots\rangle$ denotes the time average, $\vec{n}$ the unit normal of $\partial V$, and $\mathrm{d} a$ an infinitesimal surface element. The elements of $\stackrel{\leftrightarrow}{T}$ for harmonic (electric $\vec{E}$, and magnetic $\vec{H}$ ) fields are given by:

$$
\stackrel{\leftrightarrow}{T}=\left[\varepsilon_{0} \varepsilon \vec{E} \vec{E}+\mu_{0} \mu \vec{H} \vec{H}-\frac{1}{2}\left(\varepsilon_{0} \varepsilon|\vec{E}|^{2}+\mu_{0} \mu|\vec{H}|^{2}\right) \overleftrightarrow{I}\right],
$$

with $\mu$ the magnetic susceptibility of the surrounding medium, $\mu_{0}$ the magnetic susceptibility for vacuum, and $\overleftrightarrow{I}$ the unit matrix tensor. Eqn (6) is of general validity, and provides the force acting on an arbitrary particle for any illumination source. It is important to note that the fields used to calculate the force are the self-consistent fields of the problem, which means that they are a superposition of the incident and the scattered fields. Therefore, before calculating the force, one has to solve the EM fields. In most cases this condition requires extensive full numerical calculation with finite element or FDTD methods.

In the case of small spherical particles, analytical expressions can be obtained for the optical forces. ${ }^{18}$ For a dipolar particle with dimensions $a \approx \lambda$ irradiated by an arbitrary monochromatic EM wave, the time averaged radiation-induced forces that are acting on this dipole can be derived from the nonrelativistic Lorentz force: ${ }^{47}$
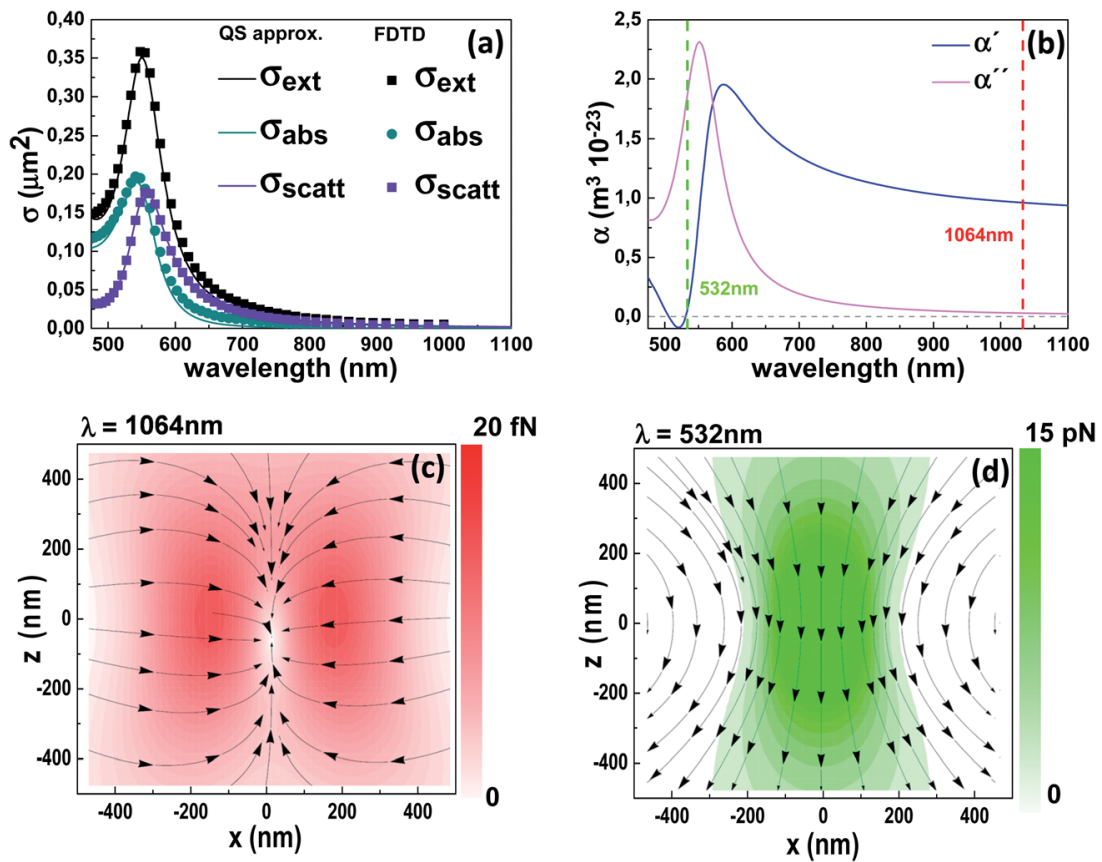

Fig. 1 (a) Extinction, absorption, and scattering cross section (in black, green, and purple, respectively) of an 80 nm diameter gold nanoparticle in water. Solid lines correspond to calculations in the extended quasistatic-approximation (using eqn (4)), and symbols correspond to FDTD simulations. The corresponding complex polarisability $\alpha(\omega)=\alpha^{\prime}(\omega)+i \alpha^{\prime \prime}(\omega)$ calculated using eqn (3) is displayed in panel (b). (c and d) Spatial distribution of the optical forces calculated through eqn (11), when a $1064 \mathrm{~nm}$ or a $532 \mathrm{~nm}$ laser is used, respectively. 


$$
\langle\vec{F}\rangle=\frac{1}{4} \varepsilon_{0} \varepsilon \alpha^{\prime} \nabla\left(\vec{E}^{*} \vec{E}\right)+\frac{1}{2} \varepsilon_{0} \varepsilon \alpha^{\prime \prime} \operatorname{Im}\left(\sum_{l} E_{l}^{*} \nabla E_{l}\right),
$$

where the subscript runs over $l=x, y, z$, and the superscript * signifies the complex conjugate. In contrast to eqn (5), the EM fields in eqn (7) are the incident fields since it is assumed that the dipole is not strong enough to disturb the incoming light. The total force can be split into two components, the gradient $\left\langle\vec{F}_{\text {grad }}\right\rangle$ and the scattering $\left\langle\vec{F}_{\text {scatt }}\right\rangle$ force:

$$
\begin{gathered}
\left\langle\vec{F}_{\text {grad }}\right\rangle=\frac{1}{4} \varepsilon_{0} \varepsilon \alpha^{\prime} \nabla\left(\vec{E}^{*} \vec{E}\right), \\
\left\langle\vec{F}_{\text {scatt }}\right\rangle=\frac{1}{2} \varepsilon_{0} \varepsilon \alpha^{\prime \prime} \operatorname{Im}\left(\sum_{l} E_{l}^{*} \nabla E_{l}\right) .
\end{gathered}
$$

The gradient force originates from the gradient of the intensity profile of the incoming laser and is proportional to the dispersive part of the complex polarisability $\left(\alpha^{\prime}\right)$. The scattering force is proportional to the dissipative part $\left(\alpha^{\prime \prime}\right)$, and represents the momentum transfer from the external radiation field to the nanoparticle by scattering and absorption. Polarisable particles are accelerated by the gradient force towards intensity maxima of the radiation field. Therefore, a tightly focused laser beam may trap a particle in 3 dimensions at its focus. However, the scattering force pushes the particle in the direction of light propagation and if the focus of the trapping laser is not tight enough, the particle may be pushed out of the trap. Stable trapping is only possible if $\left\langle\vec{F}_{\text {scatt }}\right\rangle \ll\left\langle\vec{F}_{\text {grad }}\right\rangle$, and as it can be seen from eqn (8), this will depend on the material, size and shape of the particle, as well as on the surrounding medium and the incident wavelength. We can tune the contribution of $\alpha^{\prime}$ and $\alpha^{\prime \prime}$ to $\left\langle\vec{F}_{\text {scatt }}\right\rangle$ and $\left\langle\vec{F}_{\text {grad }}\right\rangle$, respectively, with the incident wavelength of the beam. Fig. $1 \mathrm{~b}$ shows the complex polarisability of an $80 \mathrm{~nm}$ diameter gold nanoparticle in water. At long wavelengths $\left(\lambda \in[900,1000] \mathrm{nm}\right.$, marked with a red dashed line), $\alpha^{\prime}$ dominates the optical response, therefore stable 3-dimensional trapping will occur if a Gaussian laser beam is focused at those wavelengths. Fig. 1c displays the spatial distribution of the total force acting on the same gold nanoparticle as in panels (a and b), when it is illuminated with a $1064 \mathrm{~nm}$ laser, showing stable trapping at the focal point. In this case, the condition $\left\langle\vec{F}_{\text {scatt }}\right\rangle \ll$ $\left\langle\vec{F}_{\text {grad }}\right\rangle$ is satisfied. For shorter wavelengths close to the plasmon resonance $\alpha^{\prime \prime}$ dominates, leading to a strong scattering force that pushes the particle along the direction of light propagation. This effect is found for the same system as before, when a $532 \mathrm{~nm}$ laser is used (Fig. 1d). Moreover, the sign of the force (attracting or repelling) can be also tuned with the incident laser. At $\lambda \approx 540 \mathrm{~nm}$, slightly blue-shifted to the plasmon resonance, $\alpha^{\prime}$ is negative, resulting in negative (repulsive) gradient forces. As a general rule of thumb, for gold nanoparticles with dimensions $a \approx \lambda$, embedded in media with low dielectric constants, illumination with an incoming Gaussian beam "off-resonance" with the localized plasmon will enable stable trapping, while an incident wavelength "on-resonance" will result in a strong scattering force pushing the particle along the light propagation in a forward-directed force. For a precise description, simulations must be performed to determine the actual nature of the forces for a given system. In order to trap particles with laser wavelengths close to their plasmon resonance, special beam shapes such as Laguerre-Gaussian beams must be implemented..$^{48,49}$

Finally we provide expressions for the radiation-induced forces for the case of small particles $(a \approx \lambda)$ near the focus of a Gaussian laser beam that contain experimentally accessible parameters. The fields at a distance $z$ from the beam focus and at a radial position $r$ from the beam axis in cylindrical coordinates is, in the Gaussian approximation: ${ }^{50}$

$$
\begin{aligned}
\vec{E}(r, z)= & \vec{E}_{0} \sqrt{\frac{2}{\pi}} \frac{\varpi_{0}}{\varpi(z)} \exp \left[-\frac{r^{2}}{\varpi^{2}(z)}\right] \exp \left[i \frac{k r^{2}}{2 R(z)}\right] \\
& \times \exp [i k z-i \eta(z)],
\end{aligned}
$$

where

$$
\begin{gathered}
\varpi^{2}(z)=\varpi_{0}{ }^{2}\left[1+\left(\frac{z}{z_{0}}\right)^{2}\right], \\
R(z)=z\left[1+\left(\frac{z_{0}}{z}\right)^{2}\right], \\
\eta(z)=\tan ^{-1}\left(\frac{z}{z_{0}}\right) .
\end{gathered}
$$

We can define the maximum electric field amplitude in the focus as $\left|\vec{E}_{0}\right|=2 / \varpi_{0} \sqrt{Z_{0} P / \pi}$, where $Z_{0}=\sqrt{\mu_{0} / \varepsilon_{0}}$ is the impedance of free space, and $P$ is the laser power (in Watts). In the above expressions we have defined $z_{0}=\pi \varpi_{0}^{2} \sqrt{\varepsilon} / \lambda$, with the beam radius in the focus $\varpi_{0}=\lambda /(\mathrm{NA} \pi)$, and the numerical aperture of the objective, NA.

If we now combine eqn (9) with (8), we obtain analytical expression for the radial and $z$-components of the gradient and scattering forces: ${ }^{50}$

$$
\begin{gathered}
F_{\text {grad }}{ }^{r}(r, z)=-\frac{2 \varepsilon_{0}}{\pi} \alpha^{\prime}\left|\vec{E}_{0}\right|^{2} r \frac{\varpi_{0}^{2}}{\varpi^{4}} \exp \left[-\frac{2 r^{2}}{\varpi^{2}}\right] \\
F_{\text {grad }}{ }^{z}(r, z)=-\frac{\varepsilon_{0}}{\pi} \alpha^{\prime}\left|\vec{E}_{0}\right|^{2} z \frac{\varpi_{0}^{4}}{z_{0}^{2}}\left(\frac{1}{\varpi^{4}}-\frac{2 r^{2}}{\varpi^{6}}\right) \exp \left[-\frac{2 r^{2}}{\varpi^{2}}\right], \\
F_{\text {scatt }}{ }^{r}(r, z)=\frac{\varepsilon_{0}}{\pi} \alpha^{\prime \prime}\left|\vec{E}_{0}\right|^{2} r \frac{\varpi_{0}^{2}}{\varpi^{2}} \frac{k}{R} \exp \left[-\frac{2 r^{2}}{\varpi^{2}}\right] \\
F_{\text {scatt }}{ }^{2}(r, z)=\frac{\varepsilon_{0}}{\pi} \alpha^{\prime \prime}\left|\vec{E}_{0}\right|^{2} z \frac{\varpi_{0}^{2}}{\varpi^{2}}\left[k\left(1-\frac{r^{2}}{2} \frac{z^{2}-z_{0}^{2}}{\left(z^{2}+z_{0}^{2}\right)^{2}}\right)+\frac{\varpi_{0}^{2}}{z_{0} \varpi^{2}}\right] \\
\times \exp \left[-\frac{2 r^{2}}{\varpi^{2}}\right] .
\end{gathered}
$$

This model assumes a paraxial Gaussian beam focused by a lens, an approximation that breaks down for large NA values. ${ }^{51} \mathrm{To}$ provide an estimation of the accuracy of the paraxial calculations, the parameter $s=\lambda \sqrt{\varepsilon} /\left(2 \pi \varpi_{0}\right)$ is defined. For instance, average 
errors of $\sim 9.47 \%$ and $\sim 15.3 \%$ in the electric field have been predicted in ref. 51 for $s=0.20$, and $s=0.30$, respectively. The calculations for the radiation forces have corresponding uncertainties, depending on the NA chosen.

To sum up, by combining eqn (1), (4) and (9)-(11) it is possible to calculate semi-analytically the optical forces acting on metallic spheres with $a \approx \lambda$ embedded in a low refractive index medium and illuminated with a Gaussian beam. Results in Fig. 1 are obtained using these expressions.

\section{Plasmonic heating}

Associated with the absorption of radiation is the generation of heat which originates from non-radiative decay channels due to electron-electron and electron-phonon interactions. ${ }^{38}$ The resulting temperature distribution around a nanoparticle is described by the heat transfer equation, a differential equation that must be solved numerically: $:^{52}$

$$
\rho(\bar{r}) q(\bar{r}) \frac{\partial T(\bar{r}, t)}{\partial t}=\nabla \kappa(\bar{r}) \nabla T(\bar{r}, t)-Q(\bar{r}, t) .
$$

In the above expressions $\bar{r}$ and $t$ are the spatial coordinate and time; $T(\bar{r}, t)$ is the local temperature; $Q(\bar{r}, t)$ represents the optical power absorbed by the nanoparticle; and $\rho, q$ and $\kappa$ are the mass density, specific heat, and thermal conductivity, respectively. The function $Q(\bar{r}, t)=\langle\vec{j}(\bar{r}, t) \vec{E}(\bar{r}, t)\rangle_{t}$ represents an energy source originating from light dissipation in nanoparticles, with $\vec{j}(\bar{r}, t)$ the current density and $\vec{E}(\bar{r}, t)$ the stimulating electric field in the system. The solution of eqn (12) has a transient state, and after a characteristic time, the steady state is reached. ${ }^{52-55}$

The temperature increase around a small spherical particle $(a \ll \lambda)$ in the steady state can be solved analytically: ${ }^{52,53}$

$$
\Delta T(\vec{r})=\frac{V Q}{4 \pi \kappa_{0} r}
$$

with $\kappa_{0}$ the thermal conductivity of the surrounding medium, the distance from the surface of the nanoparticle, $r$, and the thermal energy: ${ }^{56}$

$$
Q=\frac{\sigma_{\mathrm{abs}} I}{V}
$$
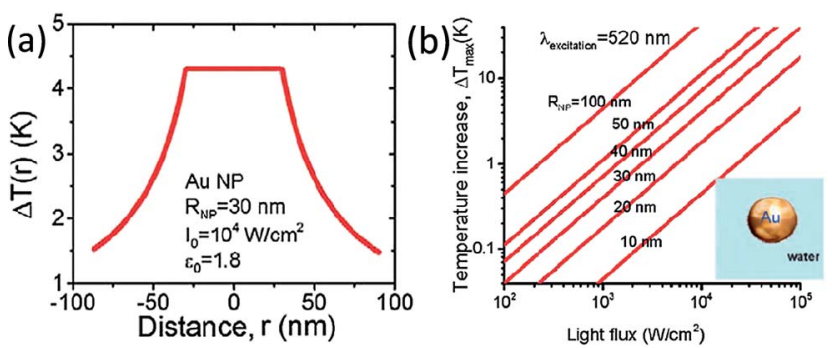

Fig. 2 (a) Temperature increase of a $60 \mathrm{~nm}$ diameter spherical gold nanoparticle in water as a function of the distance from the surface of the particle. The incident light flux is $I_{0}=10^{4} \mathrm{~W} \mathrm{~cm}^{-2}$. (b) Temperature increase for different particle sizes (as indicated in the figure) as a function of the incident light flux, illuminated at $\lambda \approx 520 \mathrm{~nm}$. Adapted and reprinted from ref. 52 with permission from Elsevier. with $I$ the power density of the incident electromagnetic wave. Note that the maximum temperature change occurs at $r=a$. As an example of the temperature distribution around a single nanoparticle, Fig. 2a shows the temperature increase for a $60 \mathrm{~nm}$ diameter spherical gold nanoparticle in water as a function of the distance from the centre of the nanoparticle. Fig. $2 \mathrm{~b}$ shows the temperature increase at the surface of nanoparticles of different radii as a function of the incident power, at $\lambda \approx 520 \mathrm{~nm}$. Combining eqn (13) and (14) shows that larger absorption cross sections lead to larger temperature changes. Thus bigger particles are more efficient heaters.

\section{Fundamental studies}

While the presence of the LSPR in metallic nanoparticles makes stable trapping more challenging, a stably trapped plasmonic particle allows for a number of fundamental phenomena to be investigated in free solution and the absence of a substrate that are not present for purely dielectric particles. A typical, basic experimental setup for studying optically trapped plasmonic nanoparticles is displayed in Fig. 3a. A laser with a wavelength longer than the LSPR of the particles to be trapped is tightly focused through a microscope objective into a droplet of a colloidal solution of the particles above a substrate. Particles will spontaneously diffuse into the focal region and be optically trapped. The same objective is used to image the trapped particle via its scattered light generated through dark-field illumination from below. Variations of this setup include additional laser sources for generating Raman scattering or fluorescence from molecules in the vicinity of the particles and have been used for several fundamental and applied studies. In the following we will discuss examples of fundamental studies concerning optical forces, the coupling of particles, and the effects of local heating.

\section{Quantification of forces}

A number of fundamental studies have focused on experimentally quantifying the optical forces experienced by plasmonic particles. In the setup described above, the optical forces are determined from histograms of the particles centre position while it is diffusing inside the trap. While being trapped the particle undergoes Brownian motion due to collisions with the water molecules, resulting in a Gaussian distribution of its position around the focal point. In most cases the trapping potential that defines the confinement of the particle can be approximated as harmonic, i.e. $U(x)=(1 / 2) K x^{2}$ where the trap stiffness $K$ is proportional to the laser intensity. If some external source displaces the bead away from the trapping position, the particle will feel a restoring force proportional to its displacement inside the trap $F(x)=-K x$. Stronger forces or stiffer traps (i.e. larger spring constants of the approximately harmonic potentials) lead to more restricted diffusion and thus narrower distributions of the centre position. Using this method, it has been demonstrated that for spherical silver nanoparticles the optical forces scale linearly with the particle volume as long as the particles are smaller than the skin depth at the trapping 

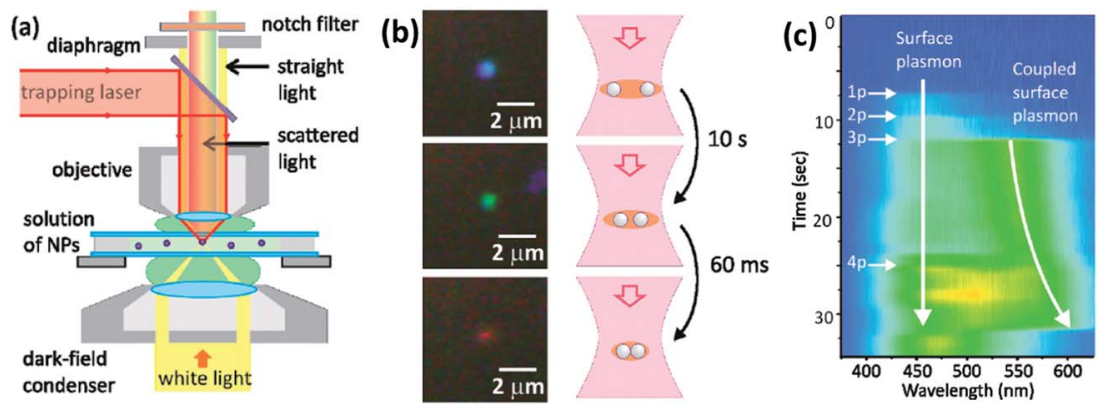

Fig. 3 (a) Typical experimental set up for trapping and observing plasmonic nanoparticles. The trapping laser is focused through a microscope objective to trap plasmonic particles in a colloidal solution. Dark field illumination is used to generate scattered light from the trapped particles, which is collected through the same objective. (b) Dark field images and schematics of trapped Ag nanoparticles displaying increasing coupling as evidenced by the apparent red shift of the scattered light. (c) Waterfall plot of scattering spectra of successively trapped Ag nanoparticles (up to 4) showing the increase in plasmonic coupling. Adapted with permission from ref. 65. Copyright 2011 American Chemical Society.

laser wavelength $(\sim 23.5 \mathrm{~nm}$ for silver at $\lambda=1064 \mathrm{~nm}) \cdot{ }^{57}$ At larger particle radii, $a$, only a surface layer is polarised by the trapping laser and the optical forces scale slower than $a^{3}$. In anisotropic particles such as gold nanorods the polarisability becomes anisotropic as well. This has two effects: firstly, for sufficiently small particles the rods will align in the trap with their long axis parallel to the trapping laser polarisation and, secondly, the optical forces do not scale linearly with the volume anymore. Even for sufficiently small particles, deviations from the linear scaling seen in spherical particles are observed. ${ }^{\mathbf{5 8 , 5 9}}$ Aligned nanorods can be rotated (or spun) by using circularly polarised lasers for trapping which transfer momentum to the trapped particles. ${ }^{58}$

\section{Coupling of plasmonic particles}

Pairs or larger aggregates of plasmonic particles exhibit novel plasmon resonances due to the coupling of the LSPRs of the individual particles. ${ }^{\mathbf{6 0 , 6 1}}$ These coupled plasmon resonances are spectrally red-shifted and give rise to large field enhancements in between the particles which are beneficial for the enhancement of low cross section processes such as Raman scattering. ${ }^{\mathbf{6 2 - 6 4}}$ Coupled plasmon resonances in an optical trap can be observed spontaneously, if two or more plasmonic particles diffuse into the focal region and are trapped together (Fig. $3 \mathrm{~b}$ and c).$^{65}$ The plasmonic coupling gives rise to a colour change in the dark field images; the closer the particles, the more the plasmon resonance is red-shifted. Coupled plasmon resonances can also be observed by bringing a trapped particle in the vicinity of a particle immobilized on a surface by moving the laser focus relative to the substrate. ${ }^{66}$ This strategy was successfully employed to observe Raman scattering from nanoparticle pairs functionalised with thiophenol molecules as a Raman analyte. ${ }^{67}$

\section{Local heating}

Plasmonic nanoparticles are well-known to act as efficient nanoscale heaters as demonstrated by their emerging application in cancer treatment. ${ }^{\mathbf{1 7 , 6 8 , 6 9}}$ Due to their small volume bubble formation is suppressed and local temperatures significantly exceeding the boiling point of the surrounding medium can be achieved. $^{\mathbf{7 0 , 7 1}}$ Even under off-resonant excitation conditions (with respect to the LSPR), as present in an optical trap, significant temperature increases can be observed. Optical trapping offers possibilities to quantify the induced temperature profiles and to study their effect on chemical and biochemical processes. In particular the absence of a substrate allows access to the intrinsic properties of the particles in solution.

Detailed studies of the temperature profiles around optically trapped gold nanoparticles have been enabled by taking advantage of a temperature-induced phase transitions in lipid bilayers from a gel to a liquid phase. ${ }^{72}$ At the transition temperature the permeability for certain dye molecules is enhanced compared to the gel phase. In addition, certain dyes partition preferentially in either the gel or the liquid phase. These two effects have been used to map the temperature profiles around optically trapped nanoparticles by observing the diffusion of dyes through a vesicle membrane and inducing local melting of a stained supported lipid bilayer. $^{70,71}$ By controlling both the distance between the bilayer/vesicle and the particle and the trapping power, it was shown that firstly, the particle surface temperature can rise by several hundred Kelvin even at moderate trapping laser powers and, secondly, the particle temperature can be controlled with a precision of a few degrees by controlling particle size and laser power.

The increase in temperature around optically trapped nanoparticles affects a number of processes in their immediate proximity. As illustrated in Fig. 4a, the hybridization of DNA molecules attached to gold nanoparticles can be monitored in an optical trap via the plasmonic coupling that accompanies the hybridization event. ${ }^{73}$ Trapping laser power dependent studies reveal that the kinetics of the DNA hybridization can be controlled by the local temperature. At higher temperatures the formation of loosely bound dimers is suppressed and consequently an overall slowing down of the binding kinetics is observed (Fig. 4b).

Particle temperature also affects the surface chemistry of the trapped particles. Ni et al. showed that optically trapped gold nanorods in an oxidizing environment undergo a temperature dependent anisotropic etching process. $^{74}$ At low trapping 
(a)

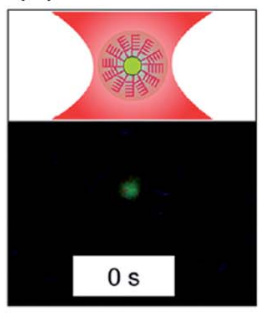

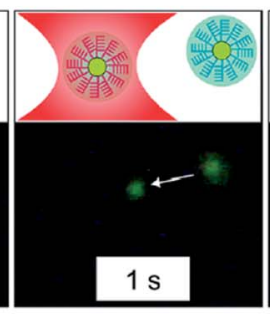

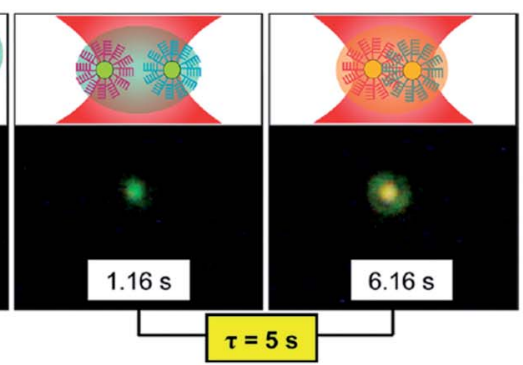

(b)

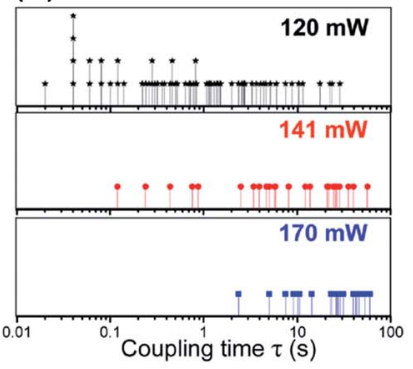

Fig. 4 (a) Dark field microscopy images and depictions of the successive trapping of DNA-functionalised Au nanoparticles and their hybridization in the trap as evidenced by plasmonic coupling. (b) Observed coupling times (i.e. "waiting times") for DNA hybridization for three different trapping powers demonstrating a slowdown of DNA hybridization kinetics at elevated temperatures due to melting of loosely bound particle dimers. Adapted with permission from ref. 73. Copyright 2013 American Chemical Society.

powers (surface temperatures) the trapped nanorods are preferentially etched from their tips since the denser ligand coverage protects the sidewalls. This is the same behaviour as observed in bulk colloidal solutions. At elevated temperatures however, the protective ligand layer on the side walls is destabilized and pre-dominant etching of the sidewalls is observed. At even higher temperature/trapping powers melting of the nanorod tips is observed, redistributing gold atoms from the tips to sidewalls, reversing the etching anisotropy again.

Lastly, particle temperature can also affect the trap stability. Ohlinger et al. showed that by successive trapping of silver nanoparticles the trap was destabilized if the red-shifted coupled plasmon resonance was close enough to the trapping laser wavelength. ${ }^{65}$ This was attributed to the combination of two effects: firstly, the coupled plasmon resonance being closer to the trapping laser wavelength shifts the force balance between gradient and scattering force towards the scattering contribution. Secondly, this also leads to more efficient plasmonic heating. Both of these effects will contribute to trap destabilization and particle release.

\section{Towards applications}

The detailed theoretical and experimental understanding of optical forces acting on plasmonic nanoparticles and the local temperatures on the surface of optically trapped particles and in their immediate vicinity enables a number of novel applications. In the following we will focus on examples for novel applications in nanofabrication, manipulation of biological and biomimetic systems, and sensing.

\section{Nanofabrication}

Nanofabrication refers to the manipulation of matter to produce nanoscale structures with useful functions enabling nanotechnology. ${ }^{75}$ There are two fundamentally different approaches for achieving this. The first is the "top-down" approach, in which beams of photons, electrons or atoms are used to carve nano- and macroscopic structures. This approach often requires many steps of preparing masks, illuminating, washing, etching, etc. and can be very time-consuming. The feature size is essentially limited by the diffraction limit and thus the wavelength of the beam used. For very small structures very large, expensive devices to produce deep-UV radiation or $\mathrm{X}$-rays need to be used, often exposing substrates to extremely harsh environments. This prohibits the use of many polymers and biomolecules on these substrates. The second approach is called "bottom-up" and uses pre-formed nanosized building blocks to build larger structures either through self-assembly or guided-assembly. Self-assembly utilizes the inherent properties of building blocks, e.g. molecules or nanoparticles, to spontaneously arrange with spacings and positions governed by interparticle and particle-substrate interactions. ${ }^{76-78}$ Inherent drawbacks of this method are a finite amount of defects and also an inability to use molecules whose interactions do not permit self-assembly. This can be overcome by using directed self-assembly, in which a structure is pre-patterned, directing the building blocks to specific locations on the substrate..$^{79}$ This method obviously introduces at least one additional step, lengthening the fabrication procedure considerably. One of the main drawbacks of these methods is that it is very difficult to use multiple types of building-blocks and most of them also lack the capability to position single nanoparticles at precise sites. ${ }^{79-82}$ Scanning force methods, with which a probe, e.g. an atomic force microscope (AFM) tip is guided over a substrate, used to pick up and place single molecules at highly defined positions, also suffer from stark drawbacks, namely the difficulties of separating the precursors from the probe tip or of using large objects. ${ }^{83,84}$

All-optical patterning of nanostructures on substrates offers the possibility to overcome all of these drawbacks. The subtle balance that can be achieved between scattering and gradient forces near the plasmon resonance enables novel approaches towards the nanopatterning of substrates. One of the first ever reports on this was published in 2002 by Hoogenboom et al. ${ }^{85} \mathrm{In}$ this report, optical tweezers were used to remove large silica particles (500-700 $\mathrm{nm}$ in radius) from a reservoir, drag them into position and attach them to a surface by shifting the focus of the optical tweezers in the axial direction of laser propagation, with a resulting accuracy of better than $80 \mathrm{~nm}$. The process was also applied for smaller nanoparticles $(7.5 \mathrm{~nm}$ radius gold core and $79 \mathrm{~nm}$ radius silica shell). Soon after, the first report of 
single gold nanoparticles fixed to a substrate appeared. In their work, Ito et al. also employed a $1064 \mathrm{~nm} \mathrm{cw}$-laser to trap single, $80 \mathrm{~nm}$ large gold nanoparticles and bring them to the substrate, where they were attached to the surface by a ns-pulsed UV-laser beam. ${ }^{86}$ Drawbacks of these processes are in the first case the need to repeatedly return to the reservoir for a new nanoparticle, and in the second case, the need for two collinearly aligned laser beams and a resulting partial melting of the nanoparticles, which may change their optical properties.

Several reports have been published over the years, detailing fabrication of micro- and macrostructures, employing optical forces and single nanoparticle precursors. An early report in 2004 by $\mathrm{Xu}$ et al. used an ultrasonic nebulizer and a cw-laser to create sub-micronsized liquid droplets, containing small gold nanoparticles and direct them to a substrate where they could aggregate to form micrometre large strips of gold. ${ }^{87}$ Zhang et al. created micronsized structures out of gold nanoparticles which aggregate out of solution due to convective currents forming at heated micronsized structures. ${ }^{88}$ Laser-induced convection was also used by Bahns et al., who could fabricate complex twodimensional patterns with a high ohmic conductance out of single gold and carbon nanoparticles; ${ }^{89,90}$ and $\mathrm{Xu}$ et al. used a femtosecond-pulsed laser to fuse gold nanodots together into sub-micronsized, low-resistive wires. ${ }^{91}$ While these reports are of great interest for certain applications, they all lack the capability to pattern substrates at the nanoscale and have no single-nanoparticle control at all.

It was not until many years later that laser beams resonant to the particle plasmon were used to attach single metallic nanoparticles to a substrate. Urban et al. reported a method to print gold nanoparticles directly out of solution onto substrates with an accuracy of better than $50 \mathrm{~nm}$ and a speed of 1 nanoparticle every $5 \mathrm{~s}$, by tuning the wavelength of the laser $(532 \mathrm{~nm})$ near to the plasmon resonance of the nanoparticles. ${ }^{92}$ An example of the accuracy is shown in Fig. 5a, in which a structure several tens of micrometres in size was printed, each nanoparticle positioned with nanometre-scale accuracy. Because the electrical charge of the preformed gold nanoparticles and the glass substrates modified with cationic polymers were of equal sign, the nanoparticles in solution did not adhere to the surface on their own. Only through the application of the scattering force of the laser could the electrostatic repulsion be overcome and the nanoparticles affixed tightly due to strong van der Waals forces. Furthermore they found that there was a trade-off between printing speed and accuracy, so that an increase in printing speed, enabled by a higher laser power, led to a reduced accuracy and vice versa. A similar method, published nearly simultaneously, used optical tweezers at $817 \mathrm{~nm}$ wavelength, and after partially removing the polymer coating from the nanoparticles, enabled Guffey et al. to attach the nanoparticles to the substrates, albeit with a larger positioning error (>100 nm).${ }^{93}$ While both of these fixation methods showed immovability of the affixed gold nanoparticles, further stability could also be provided by embedding nanoparticles into a polymer layer. ${ }^{94}$

In order to make this technique applicable to industrial fabrication, for which large substrate areas are required, the speed of printing needs to be greatly increased. Instead of trading off speed for accuracy, another possibility is parallelizing the process. By using multiple laser beams, or, as shown by Nedev et al., by using a spatial light modulator, to create a pattern of multiple laser foci, several nanoparticles can be printed simultaneously (Fig. 5b).$^{95}$ The accuracy was shown to be nearly the same as for serial nanoparticle printing but with a reported speed of 6 nanoparticles per s, an increase of about 30 times. An additional advantage of this process is that the laser pattern can be shifted and the same pattern printed repeatedly, or the pattern can be switched at high speeds enabling rapid formation of complex patterns.

One of the problems existing for all of these techniques is an apparently fundamental limitation of the minimal distance between controllably printed nanoparticles, which could not be reduced much below $300 \mathrm{~nm}$. This is most likely due to the scattered light from an already printed nanoparticle interfering with the incident light field and creating energetically favourable positions at large distances from the affixed
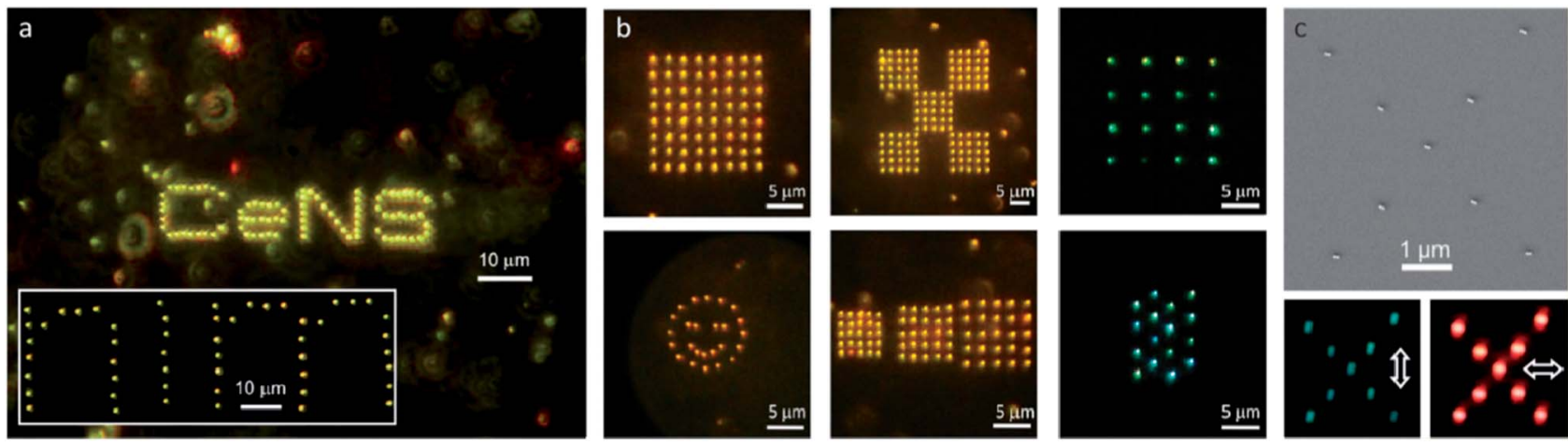

Fig. 5 Optical patterning of substrates with single nanoparticle precision. (a) $80 \mathrm{~nm}$ gold nanospheres printed by a laser beam tuned to the plasmon resonance of the nanoparticles with a positioning accuracy of better than $50 \mathrm{~nm}$. (b) Parallelization of the laser printing process through incorporation of spatial light modulators greatly increases the printing speed without loss of accuracy for both gold and silver (two right panels) $80 \mathrm{~nm}$ nanospheres. (c) Gold nanorods can be oriented on a substrate by printing with a laser beam tuned to the longitudinal plasmon resonances of the nanorods. Adapted with permission from ref. 92, 95 and 100. Copyright 2010, 2011, and 2013 American Chemical Society. 
nanoparticle. ${ }^{\mathbf{9 6}, 97}$ This drawback limits the possibilities of printed nanostructures, because most of the interparticle distances will be too large to permit plasmonic coupling between the nanoparticles. A possible approach to overcome this is to use intermediate substrates that can be reshaped after printing. By using thermo-responsive polymers, which can be stretched and then frozen in a meta-stable state, a printed nanopattern can be shrunk in size by the amount at which the polymer was previously stretched. ${ }^{98}$ A polymer with a shrinking ratio of 2.5 was used to obtain final geometries comprising $80 \mathrm{~nm}$ gold nanospheres with interparticle gaps of only 20$40 \mathrm{~nm}$. These distances could be reduced even further in the future by using polymers with higher shrinking factors.

Optical printing is not exclusive to metallic nanospheres and can be extended to nanoparticles of other shapes and materials: silver nanospheres were printed in the same way as before for gold nanospheres (Fig. 5b, two right-most panels). ${ }^{95}$ Bipyramidal nanoparticles exhibit more complex scattering spectra with a strong, aspect-ratio dependent maximum in the nearinfrared wavelength regime and were selectively printed out of a solution containing these and similarly sized nanospheres by using a laser tuned to the longitudinal resonance of the bipyramids. ${ }^{99}$ Gold nanorods, a similar structure, can be printed in much the same way. Additionally the nanorods can be oriented in specific directions, because the longitudinal axis has a preferential position in a linearly polarised beam tuned to the corresponding longitudinal resonance (Fig. 5c). Do et al. employed a two-colour optical printing scheme in which a non-resonant trapping laser was used to position and align the nanorods, while a resonant laser was used for the printing step. A spatial precision similar to the previously mentioned studies was achieved and an aligning accuracy of $17^{\circ}$ was reported, as can be seen in the SEM image of the nanorods (Fig. 5c, top panel) and by imaging the rods in a dark-field microscope and rotating a linear polariser in the emission path by $90^{\circ}$ (bottom panels).$^{\mathbf{1 0 0}}$ This application can be essential for the fabrication of optoelectronic devices and sensors. ${ }^{\mathbf{1 0 1 - 1 0 3}}$

The printing technique itself can be controlled in a way that is very gentle to the individual precursors. It was shown by Do et al. that it is possible to print nanoparticles fabricated with an attached nanopropeller made from a DNA-origami structure without destroying these hybrid nanostructures. ${ }^{\mathbf{1 0 4}}$ The major factor that needs to be controlled here is the laser-induced heating of the nanoparticles, which plays an especially large role for smaller nanoparticles. This was also reported in another paper, in which Do et al. showed they could effectively position single gold nanoparticles inside photonic crystal cavities, strongly modifying the properties of the photonics crystals. ${ }^{105}$ By fine-tuning the excitation wavelength of the laser with respect to the plasmon resonances of the nanoparticles, the factors accuracy, speed and gentleness can be adjusted to needs and specifications of the specific system.

Future improvement to all-optical printing could come from a stronger control over the incident wavelength and power, enabling a wider range of precursor nanoparticles. This, along with tailoring of beam profiles could lead to improved accuracies and printing times. ${ }^{106}$ Another approach for improving the accuracy could come by employing multiple trapping beams, which interfere together to create a complex pattern in the optical energy landscape leading to specific energy minima. ${ }^{\mathbf{9 6 , 9 7}}$ These can be controlled with great precision possibly leading to a higher printing accuracy.

A different nanofabrication strategy, enabled by optical forces and nanoscale heating, takes inspiration from scanning probe based printing techniques, where the tip of an atomic force microscope (AFM) or scanning tunnelling microscope (STM) is used to deposit single molecules or atoms in a defined pattern or to remove material from, e.g. a polymer sample, by applying mechanical force. ${ }^{\mathbf{1 0 7 , 1 0 8}}$ In the case of scanning probe based nanolithography, the tip of an AFM, for instance, is pressed against the sample and then moved in controlled direction to 'scratch' the surface of the underlying substrate. ${ }^{\mathbf{1 0 9}}$ In addition, the tip can be heated up to a certain temperature to facilitate the removal of material due to localized melting or thermal decomposition. ${ }^{\mathbf{1 1 0}}$ These methods, however, are serial by nature, because the pattern is directly "written" into sample. Patterning a large surface area with a single tip is thus timeconsuming and less applicable compared to standard top-down nanofabrication methods such as electron beam or focused ion beam lithography although the throughput rate can be increased by using a cantilever that hosts several thousand tips at the same time. ${ }^{111-113}$ Another drawback arises from the fact that the tip is controlled mechanically and that there is a physical connection between the scanning probe and the control unit which usually restricts the applicability of scanning probe microscopy methods to nanopatterning in two dimensions.

In the all optical approach that we recently introduced, heating and guiding gold nanoparticles with optical forces enables us to avoid the above functional requirement. Compared to scanning probe methods the tip is now replaced by a single gold nanoparticle which is manipulated exclusively using optical forces.

Two different cases can be distinguished for the type of nanoparticle-based lithography described above. First, the gold nanoparticle can be used to melt and thermally decompose a polymer matrix or, second, to start a controlled polymerization. ${ }^{114-117}$ An example for the first case has been shown in literature, where gold nanoparticles were embedded into a polymer film by optothermally assisted laser embossing. ${ }^{117}$ The efficiency of this process was found to be strongly dependent on the laser intensity. This is shown exemplary in Fig. $6 a-c$ for 80 $\mathrm{nm}$ gold particles heated with a $532 \mathrm{~nm}$ laser beam on a thin layer of polyvinyl alcohol (PVA). The effect of the particle heating was analysed by AFM. Only relatively moderate laser power densities below $100 \mathrm{~kW} \mathrm{~cm}{ }^{-2}$ are necessary to heat the particles sufficiently to melt the PVA $\left(\sim 228{ }^{\circ} \mathrm{C}\right)$ and to push the particles completely into the polymer film (Fig. 6b). In addition, a small rim is formed around the particle because of molten polymer that is displaced by the particle volume. If the laser power is increased further, the temperature at the particle surface can reach values up to several hundred degrees Celsius which leads to a decomposition of the polymer $\left(\sim 520{ }^{\circ} \mathrm{C}\right)$. This is shown in Fig. $6 \mathrm{c}$ where a ring is formed around a particle irradiated with a 
a)

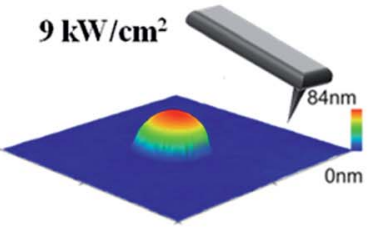

e)

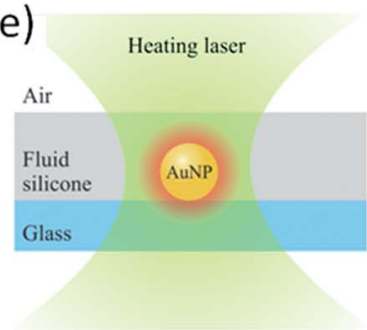

b)

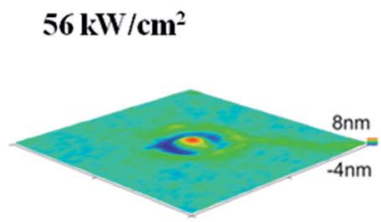

c)

$103 \mathrm{~kW} / \mathrm{cm}^{2}$

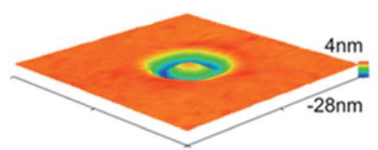

d)

\section{$220 \mathrm{~kW} / \mathrm{cm}^{2}$}

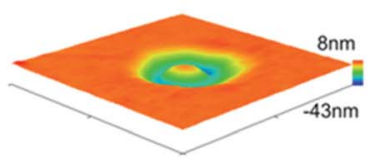

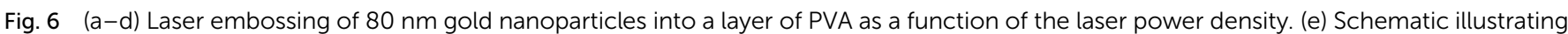

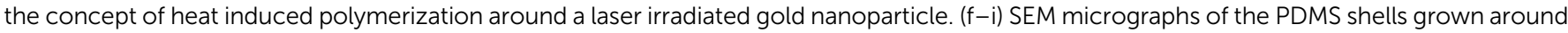

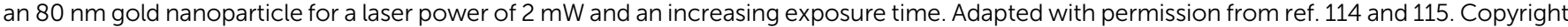
2011 and 2013 American Chemical Society.

laser power density of $220 \mathrm{~kW} \mathrm{~cm}{ }^{-2}$. One has to keep in mind, however, that the temperature reached on the particle surface also depends on the material surrounding the particle. The temperature increase in general is stronger in air compared to in a polymer network which also explains why significantly higher laser powers are necessary to start the decomposition of the material.

Instead of only melting and removing, it is also possible to use gold nanoparticles to initiate heat-induced polymerization reactions at the nanoscale. This is shown in Fig. 6e-i where a glass cover slip decorated with $80 \mathrm{~nm}$ gold nanoparticles was covered with a precursor blend of optically transparent poly(dimethylsiloxane) (PDMS) and a thermosensitive curing agent. PDMS is one of the most widely used polymers with a temperature controlled polymerization rate. ${ }^{118}$ Thermal curing takes about one day at room temperature, but can be significantly accelerated at higher temperatures.

The gold nanoparticles immersed in the PDMS blend were illuminated with a laser power of $2 \mathrm{~mW}$. Afterwards, the sample was rinsed with hexane to remove any polymer residuals that were not cross-linked and the substrate was analysed by scanning electron microscopy (SEM). As shown in Fig. 6e-i, a thin shell of PDMS was formed around the particles where the shell thickness was found to increase with increasing exposure time. The shell thickness represents therefore a direct measure of the overall particle temperature.

The heating-based methods described above can be combined further with optical forces to heat and move the nanoparticles at the same time. This could be used to control polymer decomposition and melting by pushing a strongly heated particle with a laser beam along a polymer thin-film. A schematic of the process for particle guiding in PVA is shown in Fig. 7a. Here, the radial component of the optical force exerted by the laser beam is used to guide a hot particle in the sample plane. Traces of the particle movement are formed in the PVA film and can be detected by dark-field microscopy and AFM as shown in Fig. $7 \mathrm{~b}$ and $\mathrm{c}$. The length of the tracks can be on the order of several micrometres while the width is defined by the diameter of the nanoparticle that was used to create the nanochannel. For a $40 \mathrm{~nm}$ gold nanoparticle, for example, the channel width was found to be $49 \mathrm{~nm}$ while an $80 \mathrm{~nm}$ particle produced tracks with a width of $98 \mathrm{~nm}$. These results clearly illustrate the capability of optical milling to produce nanostructures well below the diffraction limit. An important aspect to keep in mind is that the nanoparticle in this experiment is not optically trapped, but pushed by the laser. Moving and heating at the same time thus requires an appropriate adjustment of both the radial and axial component of the optical forces. The ratio between both forces must be high enough to move the particle and the amount of light absorbed by the particles must be sufficient to induce melting and decomposition of the polymer film.

The optical forces acting on a metal nanoparticle during the milling process are strongly influenced by the refractive index of the medium surrounding the nanoparticle. A polymer network has a higher refractive index than air. In the case of PVA the refractive index increases from 1.0 to 1.52 . This change has a substantial effect on the radial force acting on the particle. In air, the radial forces point towards the beam axis. Particles are therefore pulled to the centre of the laser beam, similar to optical trapping. If the refractive index is increased, the radial force component (in contrast to the axial force), which is mostly imposed by the gradient force, decreases and does in fact become negative for a medium refractive index large than 1.1. Particles interacting with the laser beam are therefore pushed out of the laser focus and along the surface of the substrate. Consequently, particles immersed in oil or a liquid polymer blend with a low viscosity can be guided over a long distance by 
a)

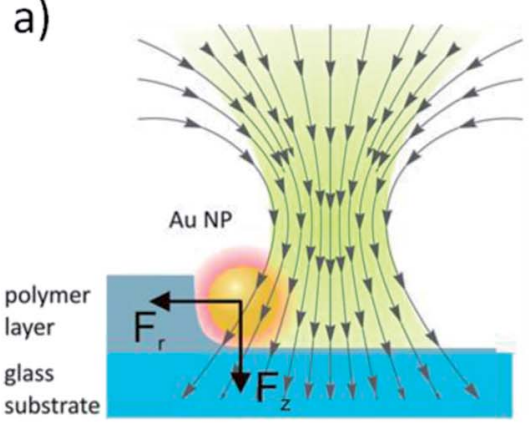

b)

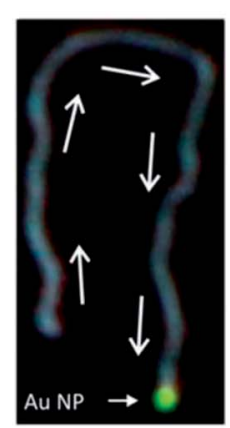

c)

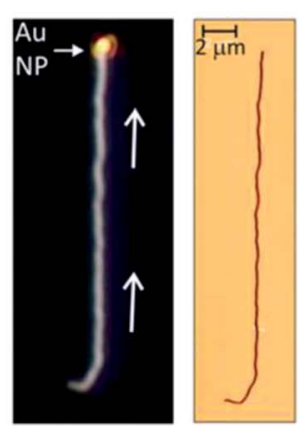

Fig. 7 (a) Schematic representation of the optical milling process inside a polymer layer. (b,c) Dark-field and AFM images of nanochannel tracks in a PVA layer produced by a single gold nanoparticle that was heated and optically guided simultaneously with laser light. Adapted with permission from ref. 114. Copyright 2011 American Chemical Society.

just steering the laser beam. If the polymer blend is thermally curable, as in the case of PDMS, this effect can be harnessed to fabricate single polymer nanowires as shown in Fig. 8a.

Here, the sample was prepared the same way as in the case of the PDMS polymerization experiment shown in Fig. 6e-i. The laser beam was focused slightly above the surface of the sample and approached from the side in order to push the particle. Upon interaction with the laser beam the polymer blend started to polymerize within milliseconds. SEM and dark field analysis of the sample revealed that PDMS nanowires with a diameter of $\sim 120 \mathrm{~nm}$ and a length of several $\mu \mathrm{m}$ were formed along the track of the particles (Fig. 8). In all cases, the particle was still visible at the end of the wire. Compared to optical milling, the formation of a polymer wire requires a more elaborate adjustment of the laser power. Too high a laser power causes the particles to heat up too strongly, which leads to an almost instantaneous curing of the polymer cast. A polymer shell that anchors the particles to the substrate is formed instantaneously a)

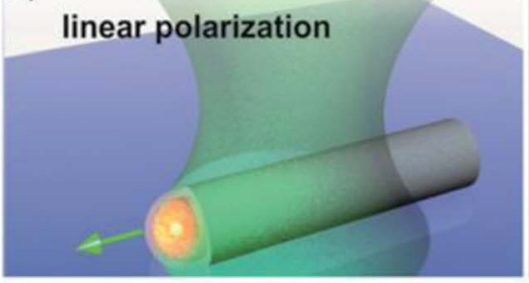

d)

left - circular polarization

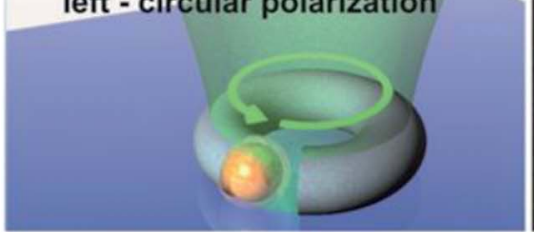

g)

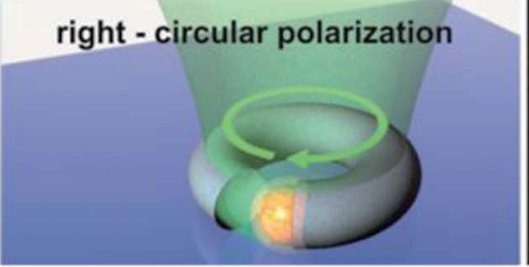

b)
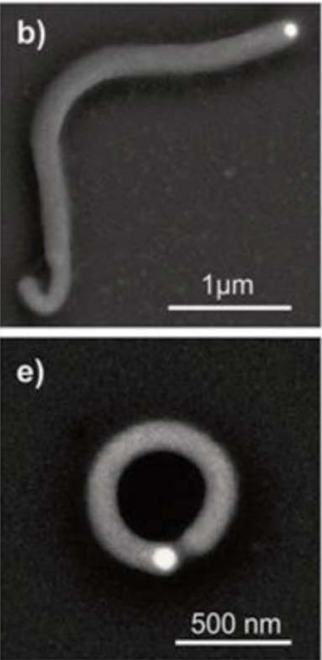

h)

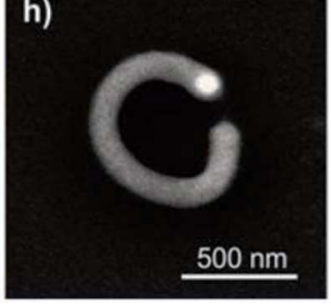

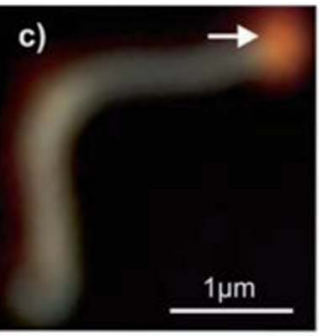
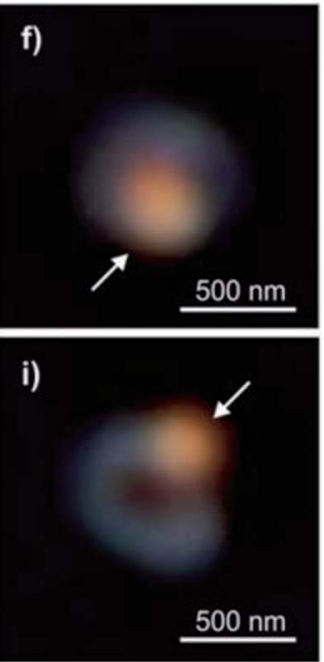

Fig. 8 ( $\mathrm{a}-\mathrm{c})$ PDMS nanowire formation by guiding a hot nanoparticle with a focused laser beam. The nanoparticle is pushed along the surface of the substrate due to optical forces that are exerted by laser light. The nanoparticle that was used to generate the wire is visible at the end of the structure. $(\mathrm{d}-\mathrm{i})$ Circularly polarised light leads to the formation of ring-shaped structures. Right-handed circularly polarised light leads to clockwise and left-handed circularly polarised light to counter-clock wise rotation due to the optical angular momentum transferred by the laser beam. Reprinted with permission from ref. 115. Copyright 2013 American Chemical Society. 
and the particles cannot be moved anymore. Intriguingly, the direction of the particle movement was found to also depend on the laser polarisation. This is because a circularly polarised laser beam for example carries a spin angular momentum which can be converted into an orbital angular momentum that is transferred to metal or even polystyrene particles upon interaction with the laser beam. ${ }^{\text {119-121 }}$ The optical angular momentum thus enables control of the particle movement direction via the laser polarisation. Linearly polarised light results in a straight line while circularly shaped structures are formed with circular polarisation. Gold nanoparticles irradiated with right-handed circularly polarised light turn clockwise while left-handed circularly polarised light leads to counterclockwise movement of the particles (Fig. 8d-i).

\section{Manipulation of biomimetic and biological systems}

The inherent characteristics of metallic nanoparticles make them prime candidates for investigating and manipulating biomimetic objects. They can be used instead of fluorescent markers, because they do not photobleach or blink. ${ }^{\mathbf{1 2 2}}$ Metallic nanoparticles larger than $40 \mathrm{~nm}$ have huge scattering cross sections and can be easily imaged directly in a dark-field microscope. ${ }^{\mathbf{1 2 3 , 1 2 4}}$ Particles smaller than this predominantly absorb light, rather than scatter it, and down to sizes of only a few nanometers can be imaged, e.g. through photothermal imaging. ${ }^{\mathbf{1 2 5 , 1 2 6}}$ In this latter method a heating laser is focused on the mainly absorbing small, metallic nanoparticles. These heat up, also elevating the temperature in their immediate surroundings. This changes the local refractive index in the medium, which can be detected by a method similar to differential interference contrast imaging. Obvious drawbacks of this method are the heat increase of the surrounding, which can perturb or even destroy biological features. ${ }^{127}$ Sensing applications are made possible because of the sensitivity of the LSPR to the surrounding matrix, and through surface-enhanced Raman scattering (SERS). ${ }^{\mathbf{1 2 8 - 1 3 0}}$ Additionally, because of their ability to efficiently convert light into heat, gold nanoparticles are excellent for investigations of dynamic processes by manipulating these processes with controllable application of heat. ${ }^{131}$

The membranes of living cells are extremely complex structures and many vital processes, such as cellular respiration, nutrient recognition and ion transport/signalling occur at or in cellular and subcellular membranes. Gold nanoparticles are excellent candidates for investigating some of these processes due to the aforementioned optical characteristics along with a low toxicity and extensive knowledge of modification of the gold surface. These capabilities in combination with the strong optical forces acting on the nanoparticle inside focused laser beams can enable even more detailed studies with highly controllable positioning of these nanometric probes.

While heating in gold nanoparticles had been investigated extensively in the past, one of the earliest reports on applying small controllable amounts of heat to investigate processes in cellular membranes was published in $2009 .^{72,132,133}$ Urban et al. used gold nanoparticles attached to the phospholipid bilayer of giant unilamellar vesicles (GUVs) to investigate the heating capabilities of the nanoparticles. By monitoring the diffusion of the gold nanoparticles, they found that not only could they induce a phase transition in the membrane, but they could also use the optical force of a laser beam to direct the nanoparticles to specific locations on the GUV membrane (Fig. 9a). The unspecific binding of the nanoparticle to the membrane somewhat reduces the applicability of this method, something that was addressed later by $\mathrm{Ba}$ et al. ${ }^{\mathbf{1 3 4}}$ Appropriate functionalisation of the nanoparticles enabled a tagging to phospholipids, which were subsequently integrated into living cell membranes. This process was monitored by imaging the diffusion of the gold nanoparticles after binding to the cell membrane (Fig. 9b). This could enable the study of specific proteins, e.g. ion channels or of lipid rafts and protein partitioning inside the cell membranes. Phospholipid membrane transitions have since been used to accurately measure the heating capacities of gold nanoparticles (see discussion of fundamental studies above).
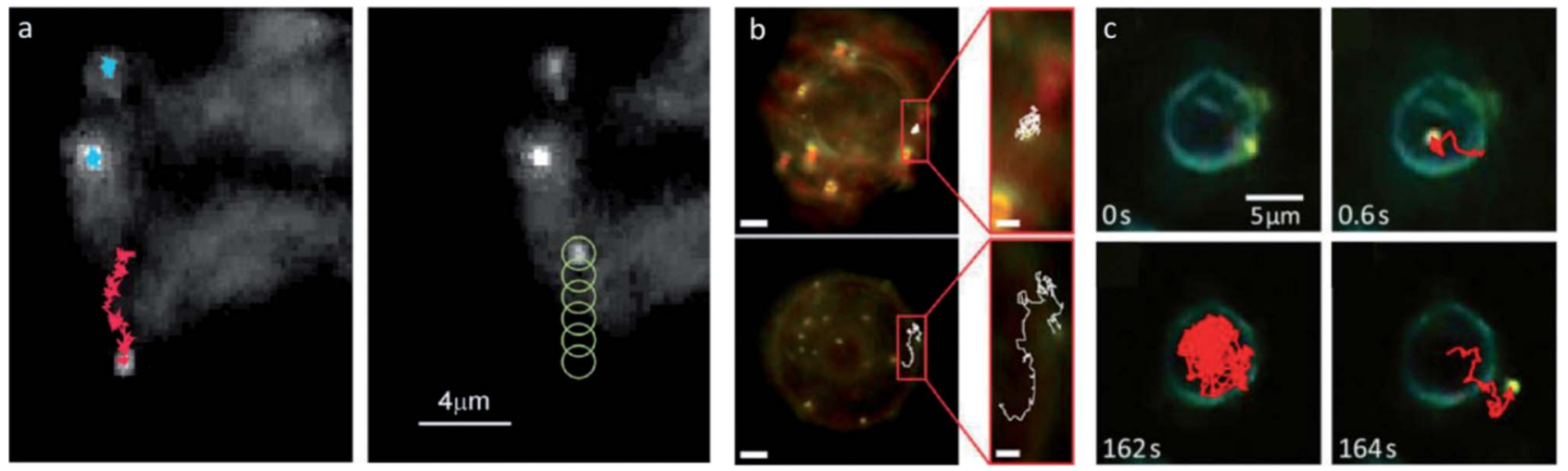

Fig. 9 Gold nanoparticles as biomimetic probes in phospholipid and cellular membranes. (a) Inducing a phase transition in a phospholipid membrane through optical heating, gold nanoparticles can be directed by optical forces to specific locations on vesicles. (b) Controlled attaching of gold nanoparticles to specific markers on the membranes of living cells can enable the study of many relevant processes occurring at or in the cell membranes. (c) The combination of the ability to controllably heat gold nanoparticles and to apply optical forces to them enables the injection of single nanoparticles through phospholipid membranes. Adapted with permission from ref. 72, 134, and 135. Copyright 2009, 2010, and 2011 American Chemical Society. 
a)

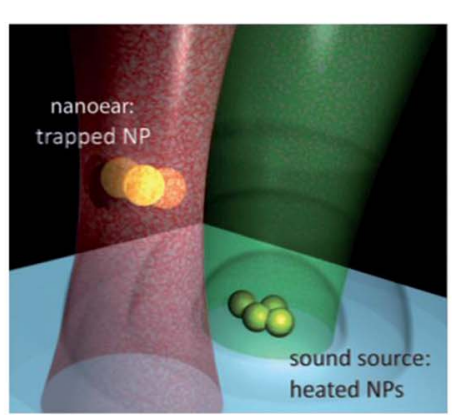

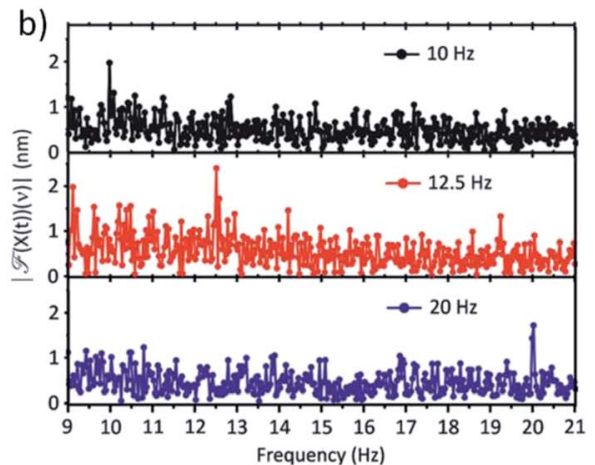

Fig. 10 (a) Schematic of the detection principle of the nano-ear: the propagating sound waves from a microscopic sound source cause a slight displacement of the particle movement in the laser trap. (b) Sound measured with an optically trapped gold nanoparticle at different frequencies between 10 and $20 \mathrm{~Hz}$. Adapted with permission from ref. 147. Copyright 2012 by The American Physical Society.

The combination of the ability to controllably heat gold nanoparticles and to apply optical forces to them, allowed Urban et al. to inject single nanoparticles through phospholipid membranes. ${ }^{135}$ The heat generated by the nanoparticles induced a phase transition and the optical forces pushed the nanoparticles inside the vesicles with the membrane detaching at the boundary between the fluid- and gel-phases. Here, the injection was monitored by tracking the diffusion of the nanoparticles (Fig. 9c). The authors were able to inject both nanoparticles that had been previously attached to the cell membranes as well as nanoparticles straight out of the solution. This represented one of the first steps towards single-cell drug delivery and targeted release of liposomal content, both of which have been studied extensively in the last couple of years and should only continue to be extended into the future. ${ }^{136-138}$

\section{Sensing}

There has been a huge progress recently in harnessing the properties of gold and silver nanoparticles for label-free sensing and spectroscopy. ${ }^{139,140}$ Gold nanoparticles in particular are ideal tools for sensing applications because they are stable in water, do not easily oxidize, and their optical properties, which can be tuned by varying their size and shape, are strongly sensitive to the dielectric properties of the medium surrounding them. ${ }^{141,142}$ This has been used to monitor the binding and release of single molecules and proteins to gold nanoparticles by measuring a shift of the plasmon resonance frequency caused by a small variation of the local refractive index. ${ }^{123,143}$

If two particles are brought in close proximity to each other the coupling between the individual plasmons can generate a strongly enhanced, localized electric field called a plasmonic 'hot-spot'. Strongly coupled plasmonic nanostructures are particularly well suited to build biosensor- and spectroscopy devices as they have been shown to enhance Raman scattering and fluorescence intensity and also shape the emission spectrum of single molecules that are positioned in the nanoantenna gap. ${ }^{144-146}$

Nanoparticles optically trapped in a liquid at room temperature are subject to significant Brownian motion. This motion is damped to a certain level by the strong gradient forces induced by the trapping laser. As discussed in the part on fundamental studies, this motion can be analysed to reveal trapping characteristics such as the shape and depth of the potential well or to analyse the fluctuations that are caused by molecules interacting with the particle in the trap.

Furthermore, this concept can also be employed to detect tiny acoustic vibrations and solvent fluctuation in liquid media. ${ }^{147}$ Detection of sound or flow which is generated by a separate sound source can be analysed by looking at the nanoparticle displacement in the direction of the sound wave propagation (Fig. 10). Utilizing optically trapped nanoparticles as a detection device by processing the nanoparticle motion in the frequency domain renders it possible to detect even faintest acoustic vibrations at sound power levels down to $-60 \mathrm{~dB}$ in liquid media, more than six orders of magnitude more sensitive than the human ear (Fig. 10b).

\section{Conclusions and outlook}

Optical trapping of plasmonic particles has significantly broadened the usefulness and applicability of optical tweezers. This is mainly due to the fact that now the plasmonic properties of metal nanoparticles including field enhancement and local heating can be employed in free solution and in absence of a substrate. Detailed theoretical understanding and precise experimental control of the optical forces and local temperatures experienced by optically trapped plasmonic particles has enabled a number of proof-of-principle experiments towards applications that will be developed further.

All optical printing with high position and orientation control has been demonstrated. This must be further scaled up and used to fabricate novel sensors and metamaterials. It is noted that the fabrication of closely spaced, plasmonically coupled nanostructures, which would provide higher field enhancements, remains a challenge. This is believed to be due to the disturbance of the electromagnetic fields by close-by already printed nanoparticles. A possibility to circumvent these problems may be the simultaneous use of several closely spaced traps or different beam shapes for the trapping and 
printing lasers. Similarly, the optothermal nanofabrication techniques discussed here could be employed to fabricate novel device structures. For this to be viable, parallelization of the reported methods needs to be demonstrated. A possible subsequent direction here would be a further downscaling of microfluidic devices by writing nanochannels into polymer layers that would function as true nanofluidic systems. A similar approach can be used to create templates for preparing metamaterials through subsequent processing.

The control of temperature in nanoscale volumes in free solution enabled by optically trapped nanoparticles offers a number of possible applications. In combination with microand nanofluidic devices a further speed-up of nanoanalytical and chemical applications in lab-on-the-chip type devices seems possible. This is based on the fact that chemical reaction rates depend exponentially on temperature. Since plasmonic particles enable the generation of local temperatures in excess of the bulk solvent boiling point, chemical reactions in nanoand microfluidic systems can be further accelerated beyond concentration effects already observed in such structures. Single-cell manipulation and drug delivery applications based on local heating will be developed further.

Last but not least, the novel sensing capabilities of optically trapped nanoparticles for acoustic fields on the micron scale offer exciting new opportunities for listening to single cells and to develop a nanoscale version of a positioning system. In the first case, novel information on cellular dynamics and interactions might be obtained on the single or few cell level, while in the latter case several trapped particles would allow triangulation of the source. It will further be possible to use this technique for the characterisation of nanoscale flow fields and thus to apply it in the analysis and characterization of micro- and nanofluidic systems.

\section{Acknowledgements}

We thank the past and present members of the Photonics and Optoelectronics Group at Ludwig-Maximilians-Universität München for their contributions to our understanding of plasmonic nanoparticles and their optical manipulation. Work on plasmonic nanoparticles in Munich is financially supported by the Deutsche Forschungsgemeinschaft (DFG) through the excellence cluster Nanosystems Initiative Munich and the collaborative research centre SFB 1032 (project A8), and the European Research Council (ERC) through the Advanced Investigator Grant HYMEM. S.C.P acknowledges financial support from the Alexander von Humboldt Foundation. F.J. acknowledges financial support from the University of Liverpool.

\section{References}

$1 \mathrm{~J}$. Kepler, De cometis libelli tres, Andre Aperger, Augsburg, 1619.

2 P. Apian, Ein kurtzer bericht der Observation und urtels des jüngst erschienenen Cometen im Weinmon und Wintermon dises 32. Jars, Ingolstadt, 1532.
3 J. C. Maxwell, A treatise on electricity and magnetism, Clarendon Press, Oxford, 1873, vol. II.

4 P. Lebedew, Ann. Phys., 1901, 311, 433-458.

5 E. F. Nichols and G. F. Hull, Ann. Phys., 1903, 317, 225-263.

6 A. Ashkin, J. M. Dziedzic, J. E. Bjorkholm and S. Chu, Opt. Lett., 1986, 11, 288-290.

7 J. R. Moffitt, Y. R. Chemla, S. B. Smith and C. Bustamante, Annu. Rev. Biochem., 2008, 77, 205-228.

8 K. C. Neuman and A. Nagy, Nat. Methods, 2008, 5, 491-505.

9 M. Dienerowitz, M. Mazilu and K. Dholakia, J. Nanophotonics, 2008, 2, 021875.

10 K. Svoboda and S. M. Block, Opt. Lett., 1994, 19, 930-932.

11 M. Pelton, J. Aizpurua and G. Bryant, Laser Photonics Rev., 2008, 2, 136-159.

12 K. L. Kelly, E. Coronado, L. L. Zhao and G. C. Schatz, J. Phys. Chem. B, 2003, 107, 668-677.

13 M. E. Stewart, C. R. Anderton, L. B. Thompson, J. Maria, S. K. Gray, J. A. Rogers and R. G. Nuzzo, Chem. Rev., 2008, 108, 494-521.

14 S. Lal, S. Link and N. J. Halas, Nat. Photonics, 2007, 1, 641648.

15 J. L. Perry and S. G. Kandlikar, Microfluid. Nanofluid., 2006, 2, 185-193.

16 G. Han, P. Ghosh and V. M. Rotello, Nanomedicine, 2007, 2, 113-123.

17 E. C. Dreaden and M. A. El-Sayed, Acc. Chem. Res., 2012, 45, 1854-1865.

18 L. Novotny and B. Hecht, Principles of Nano-Optics, Cambridge University Press, 2012.

19 P. B. Johnson and R. W. Christy, Phys. Rev. B: Solid State, 1972, 6, 4370-4379.

20 M. A. Ordal, L. L. Long, R. J. Bell, S. E. Bell, R. R. Bell, R. W. Alexander Jr and C. A. Ward, Appl. Opt., 1983, 22, 1099-1119.

21 E. D. Palik, Handbook of Optical Constants of Solids, Academic Press, 1998.

22 A. D. Rakic, A. B. Djurisic, J. M. Elazar and M. L. Majewski, Appl. Opt., 1998, 37, 5271-5283.

23 M. J. Weber, Handbook of Optical Materials, CRC Press, 2002.

24 S. G. Rodrigo, F. J. García-Vidal and L. Martín-Moreno, Phys. Rev. B: Condens. Matter Mater. Phys., 2008, 77, 075401.

25 A. Vial, A.-S. Grimault, D. Macías, D. Barchiesi and M. L. de la Chapelle, Phys. Rev. B: Condens. Matter Mater. Phys., 2005, 71, 085416.

26 K. Yee, IEEE Trans. Antennas Propag., 1966, 14, 302-307.

27 E. M. Purcell and C. R. Pennypacker, Astrophys. J., 1973, 186, 705-714.

28 A. Taflove, IEEE Trans. Electromagn. Compat., 1980, EMC-22, 191-202.

29 B. T. Draine and P. J. Flatau, J. Opt. Soc. Am. A, 1994, 11, 1491-1499.

30 J. P. Kottmann, O. J. F. Martin, D. R. Smith and S. Schultz, New J. Phys., 2000, 2, 27.

31 A. Taflove and S. C. Hagness, in Computational Electrodynamics, Artech House, Inc., Norwood, MA, 2000.

32 K. L. Shuford, M. A. Ratner and G. C. Schatz, J. Chem. Phys., 2005, 123, 114713. 
33 U. Hohenester and J. Krenn, Phys. Rev. B: Condens. Matter Mater. Phys., 2005, 72, 195429.

34 A. B. Evlyukhin and S. I. Bozhevolnyi, Phys. Rev. B: Condens. Matter Mater. Phys., 2005, 71, 134304.

35 J. Aizpurua, G. W. Bryant, L. J. Richter, F. J. García de Abajo, B. K. Kelley and T. Mallouk, Phys. Rev. B: Condens. Matter Mater. Phys., 2005, 71, 235420.

36 V. Giannini and J. A. Sánchez-Gil, J. Opt. Soc. Am. A, 2007, 24, 2822-2830.

37 V. Myroshnychenko, J. Rodriguez-Fernandez, I. PastorizaSantos, A. M. Funston, C. Novo, P. Mulvaney, L. M. LizMarzan and F. J. G. de Abajo, Chem. Soc. Rev., 2008, 37, 1792-1805.

38 U. Kreibig and M. Vollmer, Optical properties of metal clusters, Springer, Berlin, 1995.

39 J. W. Strutt, Philos. Mag. Ser. 4, 1871, 41, 107-120.

40 L. Rayleigh, Philos. Mag. Ser. 5, 1899, 47, 375-384.

41 J. D. Jackson, Klassische Elektrodynamik, Walter de Gruyter, 2006.

42 S. A. Maier, Plasmonics: Fundamentals and Applications: Fundamentals and Applications, Springer, 2007.

43 G. Mie, Ann. Phys., 1908, 330, 377-445.

44 C. Bohren and D. Huffmann, Absorption and scattering of light by small particle, John-Wiley, New York, 1983.

45 H. Kuwata, H. Tamaru, K. Esumi and K. Miyano, Appl. Phys. Lett., 2003, 83, 4625-4627.

46 M. Meier and A. Wokaun, Opt. Lett., 1983, 8, 581-583.

47 R. Loudon and S. M. Barnett, Opt. Express, 2006, 14, 1185511869.

48 N. R. Heckenberg, R. McDuff, C. P. Smith, H. RubinszteinDunlop and M. J. Wegener, Opt. Quantum Electron., 1992, 24, S951-S962.

49 M. Dienerowitz, M. Mazilu, P. J. Reece, T. F. Krauss and K. Dholakia, Opt. Express, 2008, 16, 4991-4999.

50 R. R. Agayan, F. Gittes, R. Kopelman and C. F. Schmidt, Appl. Opt., 2002, 41, 2318-2327.

51 A. Yariv, Quantum Electronics, Wiley, 1989.

52 A. O. Govorov and H. H. Richardson, Nano Today, 2007, 2, 30-38.

53 A. O. Govorov, W. Zhang, T. Skeini, H. Richardson, J. Lee and N. A. Kotov, Nanoscale Res. Lett., 2006, 1, 84-90.

54 G. Baffou, R. Quidant and F. J. García de Abajo, ACS Nano, 2010, 4, 709-716.

55 G. Baffou, R. Quidant and C. Girard, Appl. Phys. Lett., 2009, 94, 153109.

56 E. Moros, Physics of Thermal Therapy: Fundamentals and Clinical Applications, CRC Press, 2012.

57 L. Bosanac, T. Aabo, P. M. Bendix and L. B. Oddershede, Nano Lett., 2008, 8, 1486-1491.

58 L. Tong, V. D. Miljković and M. Käll, Nano Lett., 2010, 10, 268-273.

59 C. Selhuber-Unkel, I. Zins, O. Schubert, C. Sönnichsen and L. B. Oddershede, Nano Lett., 2008, 8, 2998-3003.

60 E. Prodan, C. Radloff, N. J. Halas and P. Nordlander, Science, 2003, 302, 419-422.

61 D. P. Fromm, A. Sundaramurthy, P. J. Schuck, G. Kino and W. E. Moerner, Nano Lett., 2004, 4, 957-961.
62 P. J. Schuck, D. P. Fromm, A. Sundaramurthy, G. S. Kino and W. E. Moerner, Phys. Rev. Lett., 2005, 94, 017402.

63 F. Jäckel, A. Kinkhabwala and W. Moerner, Chem. Phys. Lett., 2007, 446, 339-343.

64 M. Ringler, T. A. Klar, A. Schwemer, A. S. Susha, J. Stehr, G. Raschke, S. Funk, M. Borowski, A. Nichtl, K. Kürzinger, R. T. Phillips and J. Feldmann, Nano Lett., 2007, 7, 2753-2757.

65 A. Ohlinger, S. Nedev, A. A. Lutich and J. Feldmann, Nano Lett., 2011, 11, 1770-1774.

66 J. Prikulis, F. Svedberg, M. Käll, J. Enger, K. Ramser, M. Goksör and D. Hanstorp, Nano Lett., 2004, 4, 115-118.

67 F. Svedberg, Z. Li, H. Xu and M. Käll, Nano Lett., 2006, 6, 2639-2641.

68 C. Loo, A. Lin, L. Hirsch, M.-H. Lee, J. Barton, N. Halas, J. West and R. Drezek, Technol. Cancer Res. Treat., 2004, 3, 33-40.

69 D. P. O'Neal, L. R. Hirsch, N. J. Halas, J. D. Payne and J. L. West, Cancer Lett., 2004, 209, 171-176.

70 A. Kyrsting, P. M. Bendix, D. G. Stamou and L. B. Oddershede, Nano Lett., 2011, 11, 888-892.

71 P. M. Bendix, S. N. S. Reihani and L. B. Oddershede, ACS Nano, 2010, 4, 2256-2262.

72 A. S. Urban, M. Fedoruk, M. R. Horton, J. O. Rädler, F. D. Stefani and J. Feldmann, Nano Lett., 2009, 9, 2903-2908.

73 L. Osinkina, S. Carretero-Palacios, J. Stehr, A. A. Lutich, F. Jäckel and J. Feldmann, Nano Lett., 2013, 13, 3140-3144.

74 W. Ni, H. Ba, A. A. Lutich, F. Jäckel and J. Feldmann, Nano Lett., 2012, 12, 4647-4650.

75 G. A. Ozin, K. Hou, B. V. Lotsch, L. Cademartiri, D. P. Puzzo, F. Scotognella, A. Ghadimi and J. Thomson, Mater. Today, 2009, 12, 12-23.

76 D. Han, S. Pal, J. Nangreave, Z. Deng, Y. Liu and H. Yan, Science, 2011, 332, 342-346.

77 R. Schreiber, J. Do, E.-M. Roller, T. Zhang, V. J. Schüller, P. C. Nickels, J. Feldmann and T. Liedl, Nat. Nanotechnol., 2014, 9, 74-78.

78 E. V. Shevchenko, D. V. Talapin, N. A. Kotov, S. O'Brien and C. B. Murray, Nature, 2006, 439, 55-59.

79 T. Kraus, L. Malaquin, H. Schmid, W. Riess, N. D. Spencer and H. Wolf, Nat. Nanotechnol., 2007, 2, 570-576.

80 A. M. Hung, C. M. Micheel, L. D. Bozano, L. W. Osterbur, G. M. Wallraff and J. N. Cha, Nat. Nanotechnol., 2010, 5, 121-126.

81 T. I. Lee, W. J. Choi, K. J. Moon, J. H. Choi, J. P. Kar, S. N. Das, Y. S. Kim, H. K. Baik and J. M. Myoung, Nano Lett., 2010, 10, 1016-1021.

82 H. M. Saavedra, T. J. Mullen, P. Zhang, D. C. Dewey, S. A. Claridge and P. S. Weiss, Rep. Prog. Phys., 2010, 73, 036501.

83 T. B. Vu, N. Garcia and K. Dransfeld, Nanosources and Manipulation of Atoms Under High Fields and Temperatures: Applications, Springer, 1993.

84 E. M. Puchner, S. K. Kufer, M. Strackharn, S. W. Stahl and H. E. Gaub, Nano Lett., 2008, 8, 3692-3695.

85 J. P. Hoogenboom, D. L. J. Vossen, C. Faivre-Moskalenko, M. Dogterom and A. van Blaaderen, Appl. Phys. Lett., 2002, 80, 4828-4830. 
86 S. Ito, H. Yoshikawa and H. Masuhara, Appl. Phys. Lett., 2002, 80, 482-484.

87 J. Xu, J. Drelich and E. M. Nadgorny, Langmuir, 2004, 20, 1021-1025.

88 Y. Zhang, C. Gu, A. M. Schwartzberg, S. Chen and J. Z. Zhang, Phys. Rev. B: Condens. Matter Mater. Phys., 2006, 73, 165405.

89 J. T. Bahns, S. K. R. S. Sankaranarayanan, N. C. Giebink, H. Xiong and S. K. Gray, Adv. Mater., 2012, 24, OP242OP246.

90 J. T. Bahns, S. K. R. S. Sankaranarayanan, S. K. Gray and L. Chen, Phys. Rev. Lett., 2011, 106, 095501.

91 B.-B. Xu, R. Zhang, H. Wang, X.-Q. Liu, L. Wang, Z.-C. Ma, Q.-D. Chen, X.-Z. Xiao, B. Han and H.-B. Sun, Nanoscale, 2012, 4, 6955-6958.

92 A. S. Urban, A. A. Lutich, F. D. Stefani and J. Feldmann, Nano Lett., 2010, 10, 4794-4798.

93 M. J. Guffey and N. F. Scherer, Nano Lett., 2010, 10, 43024308.

94 L. Ling, H.-L. Guo, X.-L. Zhong, L. Huang, J.-F. Li, L. Gan and Z.-Y. Li, Nanotechnology, 2012, 23, 215302.

95 S. Nedev, A. S. Urban, A. A. Lutich and J. Feldmann, Nano Lett., 2011, 11, 5066-5070.

96 Z. Yan, U. Manna, W. Qin, A. Camire, P. Guyot-Sionnest and N. F. Scherer, J. Phys. Chem. Lett., 2013, 4, 2630-2636.

97 Z. Yan, R. A. Shah, G. Chado, S. K. Gray, M. Pelton and N. F. Scherer, ACS Nano, 2013, 7, 1790-1802.

98 A. S. Urban, M. Fedoruk, S. Nedev, A. Lutich, T. Lohmüller and J. Feldmann, Advanced Optical Materials, 2013, 1, 123127.

99 M. J. Guffey, R. L. Miller, S. K. Gray and N. F. Scherer, Nano Lett., 2011, 11, 4058-4066.

100 J. Do, M. Fedoruk, F. Jäckel and J. Feldmann, Nano Lett., 2013, 13, 4164-4168.

101 Y. Cui and C. Lieber, Science, 2001, 291, 851-853.

102 Y. Cui, Q. Wei, H. Park and C. M. Lieber, Science, 2001, 293, 1289-1292.

103 C. Hrelescu, T. K. Sau, A. L. Rogach, F. Jäckel, G. Laurent, L. Douillard and F. Charra, Nano Lett., 2011, 11, 402-407.

104 J. Do, R. Schreiber, A. A. Lutich, T. Liedl, J. RodríguezFernández and J. Feldmann, Nano Lett., 2012, 12, 50085013.

105 J. Do, K. N. Sediq, K. Deasy, D. M. Coles, J. RodríguezFernández, J. Feldmann and D. G. Lidzey, Advanced Optical Materials, 2013, 1, 946-951.

106 A. Jesacher, A. Schwaighofer, S. Fürhapter, C. Maurer, S. Bernet and M. Ritsch-Marte, Opt. Express, 2007, 15, 5801-5808.

107 R. D. Piner, J. Zhu, F. Xu, S. Hong and C. A. Mirkin, Science, 1999, 283, 661-663.

108 G.-Y. Liu, S. Xu and Y. Qian, Acc. Chem. Res., 2000, 33, 457466.

109 M. R. Vanlandingham, S. H. McKnight, G. R. Palmese, J. R. Elings, X. Huang, T. A. Bogetti, R. F. Eduljee and J. W. Gillespie, J. Adhes., 1997, 64, 31-59.

110 H. J. Mamin and D. Rugar, Appl. Phys. Lett., 1992, 61, 10031005.
111 M. A. McCord, J. Vac. Sci. Technol., B: Microelectron. Nanometer Struct.-Process., Meas., Phenom., 1997, 15, 2125-2129.

112 J. Melngailis, J. Vac. Sci. Technol., B: Microelectron. Process. Phenom., 1987, 5, 469-495.

113 K. Salaita, Y. Wang, J. Fragala, R. A. Vega, C. Liu and C. A. Mirkin, Angew. Chem., 2006, 118, 7378-7381.

114 M. Fedoruk, A. A. Lutich and J. Feldmann, ACS Nano, 2011, 5, 7377-7382.

115 M. Fedoruk, M. Meixner, S. Carretero-Palacios, T. Lohmüller and J. Feldmann, ACS Nano, 2013, 7, 7648-7653.

116 M. B. Cortie, N. Harris and M. J. Ford, Phys. Rev. B: Condens. Matter Mater. Phys., 2007, 394, 188-192.

117 A. G. Skirtach, D. G. Kurth and H. Möhwald, Appl. Phys. Lett., 2009, 94, 093106.

118 A. A. S. Bhagat, P. Jothimuthu and I. Papautsky, Lab Chip, 2007, 7, 1192-1197.

119 M. E. J. Friese, T. A. Nieminen, N. R. Heckenberg and H. Rubinsztein-Dunlop, Nature, 1998, 394, 348-350.

120 T. A. Nieminen, A. B. Stilgoe, N. R. Heckenberg and H. Rubinsztein-Dunlop, J. Opt. A: Pure Appl. Opt., 2008, 10, 115005.

121 S. H. Simpson and S. Hanna, J. Opt. Soc. Am. A, 2009, 26, 625-638.

122 D. Nagesha, G. Laevsky, P. Lampton, R. Banyal, C. Warner, C. DiMarzio and S. Sridhar, Int. J. Nanomed., 2007, 2, 813-819.

123 G. Raschke, S. Kowarik, T. Franzl, C. Sönnichsen, T. A. Klar, J. Feldmann, A. Nichtl and K. Kürzinger, Nano Lett., 2003, 3, 935-938.

124 C. Loo, A. Lowery, N. Halas, J. West and R. Drezek, Nano Lett., 2005, 5, 709-711.

125 D. Boyer, P. Tamarat, A. Maali, B. Lounis and M. Orrit, Science, 2002, 297, 1160-1163.

126 A. Gaiduk, P. V. Ruijgrok, M. Yorulmaza and M. Orrit, Chem. Sci., 2010, 1, 343-350.

127 L. Cognet, C. Tardin, D. Boyer, D. Choquet, P. Tamarat and B. Lounis, Proc. Natl. Acad. Sci. U. S. A., 2003, 100, 1135011355.

128 J. N. Anker, W. P. Hall, O. Lyandres, N. C. Shah, J. Zhao and R. P. Van Duyne, Nat. Mater., 2008, 7, 442-453.

129 A. Dahlin, M. Zach, T. Rindzevicius, M. Kall, D. S. Sutherland and F. Hook, J. Am. Chem. Soc., 2005, 127, 5043-5048.

130 R. A. Alvarez-Puebla and L. M. Liz-Marzán, Small, 2010, 6, 604-610.

131 A. G. Skirtach, C. Dejugnat, D. Braun, A. S. Susha, A. L. Rogach, W. J. Parak, H. Mohwald and G. B. Sukhorukov, Nano Lett., 2005, 5, 1371-1377.

132 S. Jain, D. G. Hirst and J. M. O'Sullivan, Br. J. Radiol., 2012, 85, 101-113.

133 H. H. Richardson, M. T. Carlson, P. J. Tandler, P. Hernandez and A. O. Govorov, Nano Lett., 2009, 9, 1139-1146.

134 H. Ba, J. Rodríguez-Fernández, F. D. Stefani and J. Feldmann, Nano Lett., 2010, 10, 3006-3012.

135 A. S. Urban, T. Pfeiffer, M. Fedoruk, A. A. Lutich and J. Feldmann, ACS Nano, 2011, 5, 3585-3590. 
136 M. Delcea, N. Sternberg, A. M. Yashchenok, R. Georgieva, H. Bäumler, H. Möhwald and A. G. Skirtach, ACS Nano, 2012, 6, 4169-4180.

137 J. He, Z. Wei, L. Wang, Z. Tomova, T. Babu, C. Wang, X. Han, J. T. Fourkas and Z. Nie, Angew. Chem., Int. Ed., 2013, 52, 2463-2468.

138 D. Volodkin, A. Skirtach and H. Möhwald, Polym. Int., 2012, 61, 673-679.

139 K. A. Willets and R. P. Van Duyne, Annu. Rev. Phys. Chem., 2007, 58, 267-297.

140 N. J. Halas, S. Lal, W.-S. Chang, S. Link and P. Nordlander, Chem. Rev., 2011, 111, 3913-3961.

141 C. Sönnichsen, S. Geier, N. E. Hecker, G. von Plessen, J. Feldmann, H. Ditlbacher, B. Lamprecht, J. R. Krenn, F. R. Aussenegg, V. Z.-H. Chan, J. P. Spatz and M. Möller, Appl. Phys. Lett., 2000, 77, 2949-2951.
142 M. Hu, C. Novo, A. Funston, H. Wang, H. Staleva, S. Zou, P. Mulvaney, Y. Xia and G. V. Hartland, J. Mater. Chem., 2008, 18, 1949-1960.

143 I. Ament, J. Prasad, A. Henkel, S. Schmachtel and C. Sönnichsen, Nano Lett., 2012, 12, 10921095.

144 J. P. Camden, J. A. Dieringer, Y. Wang, D. J. Masiello, L. D. Marks, G. C. Schatz and R. P. Van Duyne, J. Am. Chem. Soc., 2008, 130, 12616-12617.

145 A. Bek, R. Jansen, M. Ringler, S. Mayilo, T. A. Klar and J. Feldmann, Nano Lett., 2008, 8, 485-490.

146 M. Ringler, A. Schwemer, M. Wunderlich, A. Nichtl, K. Kürzinger, T. A. Klar and J. Feldmann, Phys. Rev. Lett., 2008, 100, 203002.

147 A. Ohlinger, A. Deak, A. A. Lutich and J. Feldmann, Phys. Rev. Lett., 2012, 108, 018101. 